\title{
Nucleophilic Substitution Reactions of Pyranose Polytosylates
}

Ross P. McGeary, ${ }^{*}{ }^{\dagger,}$ Sara Rasoul Amini, ${ }^{\star}$ Vincent W.S. Tang, ${ }^{\ddagger}$ and Istvan Toth, ${ }^{\dagger \neq}$

${ }^{\dagger}$ Chemistry Department, School of Molecular and Microbial Sciences, The University of

Queensland, Brisbane, Qld 4072, Australia.

${ }^{\ddagger}$ School of Pharmacy, The University of Queensland, Brisbane, Qld 4072, Australia.

r.mcgeary@mailbox.uq.edu.au

Table of Contents

\begin{tabular}{|c|c|}
\hline Compound & Page \\
\hline Methyl $\beta$-D-Xylopyranoside (5) & S-2 \\
\hline Methyl 2,3,4-Tris-( $O$-4-toluenesulfonyl)- $\beta$-D-xylopyranoside (6) & S-3 \\
\hline Methyl 4-O-Benzoyl-2,3-bis-(O-4-toluenesulfonyl)- $\alpha$-L-arabinopyranoside (7) & S-6 \\
\hline $\begin{array}{l}\text { Methyl 4-Azido-2,3-Bis-( } O \text {-4-toluenesulfonyl)-4-deoxy- } \alpha \text {-L-arabinopyranoside } \\
\text { (8) }\end{array}$ & S-10 \\
\hline Methyl 2,3-Bis-(O-4-toluenesulfonyl)- $\alpha$-L-arabinopyranoside (9) & S-13 \\
\hline Methyl 2,3,4-Tris-( $O$-methanesulfonyl)- $\beta$-D-xylopyranoside (10) & S-16 \\
\hline Methy $\beta$-L-Arabinopyranoside (12) & S-16 \\
\hline Methyl 2,3,4-Tris-( $O$-4-toluenesulfonyl)- $\beta$-L-arabinopyranoside (13) & S-17 \\
\hline Methyl 4-O-Benzoyl-2,3-bis-(O-4-toluenesulfonyl)- $\alpha$-D-xylopyranoside (14) & S-20 \\
\hline Methyl 4-Azido-2,3-bis-( $O$-4-toluenesulfonyl)-4-deoxy $\alpha$-D-xylopyranoside (15) & S-24 \\
\hline Methyl 2,3-Bis-(O-4-toluenesulfonyl)- $\alpha$-D-xylopyranoside (16) & S-27 \\
\hline
\end{tabular}




\begin{tabular}{|c|c|}
\hline Methyl $\alpha$-D-Ribopyranoside (18) & S-30 \\
\hline Methyl 2,3,4-Tris-( $O$-4-toluenesulfonyl)- $\alpha$-D-ribopyranoside (19) & S-30 \\
\hline Methyl 4-Benzoyl-2,3-bis-( $O$-4-toluenesulfonyl)- $\beta$-L-lyxopyranoside (20) & S-34 \\
\hline Methyl 4-Azido-2,3-bis- $(O-4$-toluenesulfonyl)-4-deoxy- $\beta$-L-lyxopyranoside (21) & S-37 \\
\hline Methyl 2,3-Bis- $(O-4$-toluenesulfonyl)- $\beta$-L-lyxopyranoside (22) & S-40 \\
\hline Methyl $\alpha$-D-Lyxopyranoside (24) & S-43 \\
\hline Methyl 2,3,4-Tris-( $O$-4-toluenesulfonyl)- $\alpha$-D-lyxopyranoside (25) & S-43 \\
\hline $\begin{array}{l}\text { 3R,6S-3-Benzoyloxy-6-methoxy-4-(4-toluenesulfonyloxy)-3,6-dihydro- } 2 H \text { - } \\
\text { pyran (26) }\end{array}$ & S-47 \\
\hline
\end{tabular}

\section{Experimental Section}

Methyl $\beta$-D-Xylopyranoside (5). A mixture of D-xylose (4) (20.0 g, $133 \mathrm{mmol})$ and ionexchange resin (Amberlite ${ }^{\circledR}$ IRA-120, $20.0 \mathrm{~g}$ ) in anhydrous methanol (250 ml) was heated under reflux overnight. The cooled mixture was filtered and evaporated to a mixture of solids and oil. This was taken up in $200 \mathrm{ml}$ hot ethyl acetate. Crystals formed on cooling, and the crystals were allowed to digest for three days before collecting, yielding $10.8 \mathrm{~g}$. Analysis of the product by ${ }^{1} \mathrm{H}$ and ${ }^{13} \mathrm{C}$ NMR spectroscopy showed that it consisted of a 2:1 mixture of $\beta$ - and $\alpha$-anomers. A second recrystallization from ethanol gave the pure $\beta$-anomer (5), as a white powder $(5.74 \mathrm{~g}, 26 \%)$, m.p. $154.5-155.5^{\circ} \mathrm{C}$ (lit. m.p. $\left.{ }^{1} 156-157{ }^{\circ} \mathrm{C}\right) . R_{f}\left(3: 2: 1 \mathrm{CHCl}_{3} / \mathrm{EtOAc} / \mathrm{MeOH}\right): 0.23 .[\alpha]_{\mathrm{D}}-65.6\left(c 0.7, \mathrm{H}_{2} \mathrm{O}\right)\left(\right.$ lit. $^{2}$ $\left.[\alpha]_{\mathrm{D}}-65.3\left(c 1.0, \mathrm{H}_{2} \mathrm{O}\right)\right) .{ }^{1} \mathrm{H}$ NMR $\left.\left(500 \mathrm{MHz},\left(\mathrm{CD}_{3}\right)_{2} \mathrm{SO}\right)\right)$ 2.89-2.96 (m, 1H, H2); 3.03 (t, $1 \mathrm{H}, J=10.8 \mathrm{~Hz}, \mathrm{H} 5 \mathrm{a}) ; 3.06-3.11(\mathrm{~m}, 1 \mathrm{H}, \mathrm{H} 4) ; 3.24-3.29(\mathrm{~m}, 1 \mathrm{H}, \mathrm{H} 3) ; 3.35(\mathrm{~s}, 3 \mathrm{H}$, OMe); 3.70 (dd, 1H, $J=5.3,11.2 \mathrm{~Hz}, \mathrm{H} 5 \mathrm{e}) ; 4.00$ (d, 1H, $J=7.6 \mathrm{~Hz}, \mathrm{H1}$ ); 4.89 (d, 1H, $J$ 
$=4.8 \mathrm{~Hz}, \mathrm{OH}) ; 4.91(\mathrm{~d}, 1 \mathrm{H}, J=5.1 \mathrm{~Hz}, \mathrm{OH}) ; 5.00(\mathrm{~d}, 1 \mathrm{H}, J=4.9 \mathrm{~Hz}, \mathrm{OH}) .{ }^{13} \mathrm{C} \mathrm{NMR}$ (125 MHz, $\left.\left.\left(\mathrm{CD}_{3}\right)_{2} \mathrm{SO}\right)\right)$ 56.5, 66.2, 70.2, 73.8, 77.1, 105.3.

Methyl 2,3,4-Tris-( $O$-4-toluenesulfonyl)- $\beta$-D-xylopyranoside (6). Methyl $\beta$-Dxylopyranoside (5) $(500 \mathrm{mg}, 3.05 \mathrm{mmol})$ was added to a stirred, chilled $\left(0{ }^{\circ} \mathrm{C}\right)$ solution of 4-toluenesulfonyl chloride $(2.32 \mathrm{~g}, 12.2 \mathrm{mmol})$ in pyridine $(10 \mathrm{ml})$. After standing for 7 days, the mixture was poured onto ice water $(50 \mathrm{ml})$. The product separated as a sticky mass. The mixture was extracted into $\mathrm{CH}_{2} \mathrm{Cl}_{2}(3 \times 50 \mathrm{ml})$ and the combined extracts were washed with $5 \% \mathrm{HCl}(3 \times 100 \mathrm{ml})$, saturated $\mathrm{NaHCO}_{3}(100 \mathrm{ml})$ and brine $(50 \mathrm{ml})$, then dried $\left(\mathrm{MgSO}_{4}\right)$ and evaporated to a colorless foam. The product was dissolved in a little methanol and slow evaporation of the solvent yielded the tritosylate $(\mathbf{6})$ as colorless crystals $(1.90 \mathrm{~g}, 99 \%)$, m.p. $137.5-138.5^{\circ} \mathrm{C}$ (lit. ${ }^{3}$ m.p. $\left.140-141{ }^{\circ} \mathrm{C}\right) . \quad R_{f}(1: 1$

EtOAc/hexane): 0.34. $[\alpha]_{\mathrm{D}}-32.2\left(c 1.0, \mathrm{CHCl}_{3}\right)\left(\right.$ lit. $^{4}[\alpha]_{\mathrm{D}}-33.7\left(c 1.0, \mathrm{CHCl}_{3}\right)$. ES-MS: $627(\mathrm{MH})^{+}, 644\left(\mathrm{M}+\mathrm{NH}_{4}\right)^{+}, 649(\mathrm{M}+\mathrm{Na})^{+} .{ }^{1} \mathrm{H} \mathrm{NMR}(500 \mathrm{MHz}, \mathrm{CDCl}) 2.44(\mathrm{~s}, 3 \mathrm{H}$, Me); 2.45 (s, 3H, Me); 2.46 (s, 3H, Me); 3.14 (s, 3H, OMe); 3.48 (ddd, 1H, $J=0.8,4.6$, $12.9 \mathrm{~Hz}, \mathrm{H} 5 \mathrm{e}) ; 4.06(\mathrm{dd}, 1 \mathrm{H}, J=3.1,12.9 \mathrm{~Hz}, \mathrm{H} 5 \mathrm{a}) ; 4.30(\mathrm{ddd}, 1 \mathrm{H}, J=0.5,3.4,4.9 \mathrm{~Hz}$, H2); 4.36 (dt, 1H, $J=3.3,4.8, \mathrm{H} 4) ; 4.39$ (d, 1H, $J=3.3 \mathrm{~Hz}, \mathrm{H1}$ ); 4.71 (t, $1 \mathrm{H}, J=5.0$, $\mathrm{H} 3) ;$ 7.31-7.36 (m, 6H, Ar-H); 7.70-7.75 (m, 6H, Ar-H). ${ }^{13} \mathrm{C}$ NMR (125 MHz, CDCl $)$ 21.5, 21.6, 21.7, 56.0, 58.8, 72.2, 73.0, 73.6, 99.0, 128.0, 128.1, 128.3, 129.7, 129.8., 129.9, 132.4, 132.6, 133.3, 145.0, 145.3, 145.4. HRMS (TOF) calcd for $\mathrm{C}_{27} \mathrm{H}_{31} \mathrm{O}_{11} \mathrm{~S}_{3}$ $(\mathrm{M}+\mathrm{H})^{+}$627.1029, found 627.1043. 


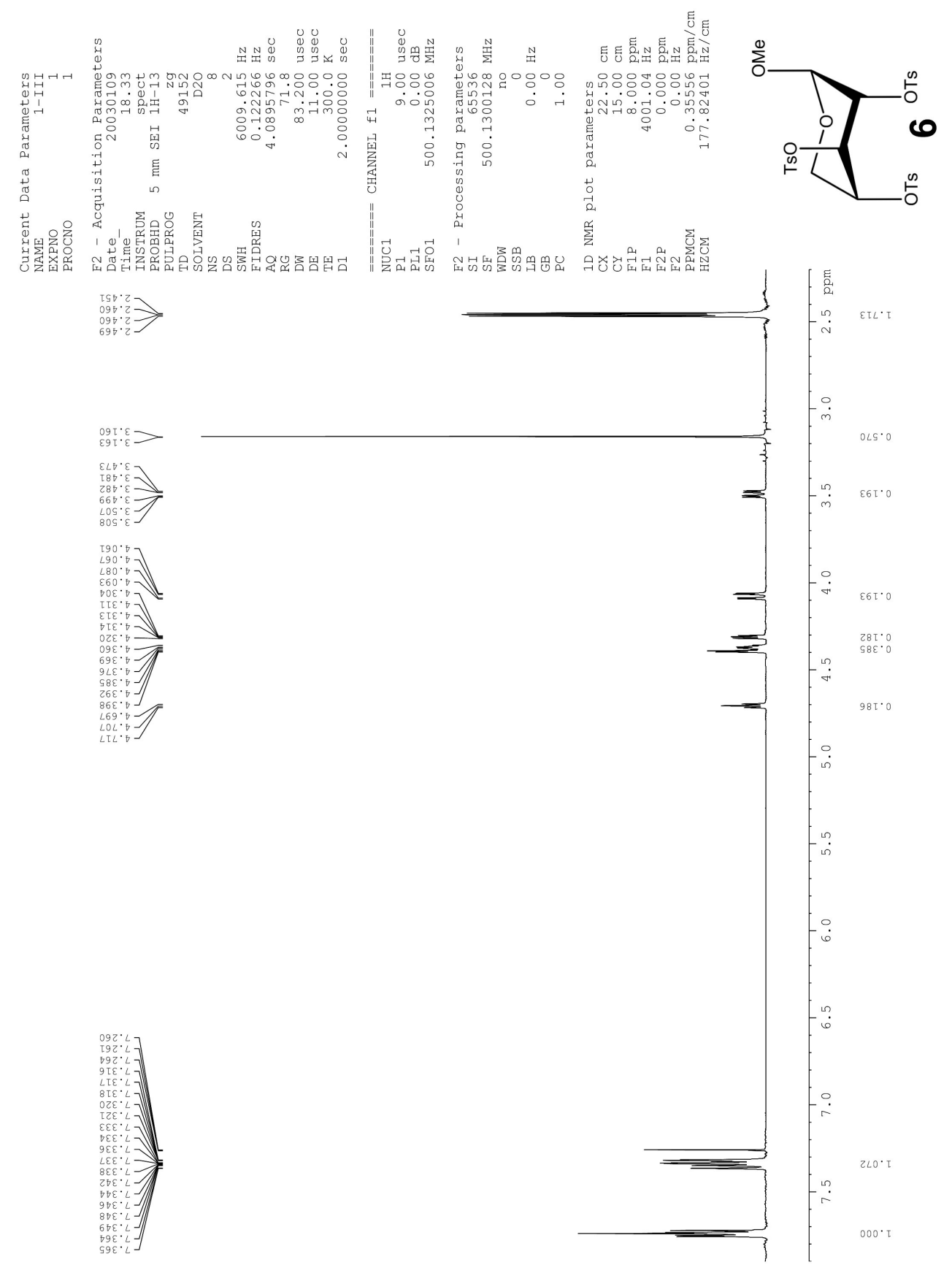



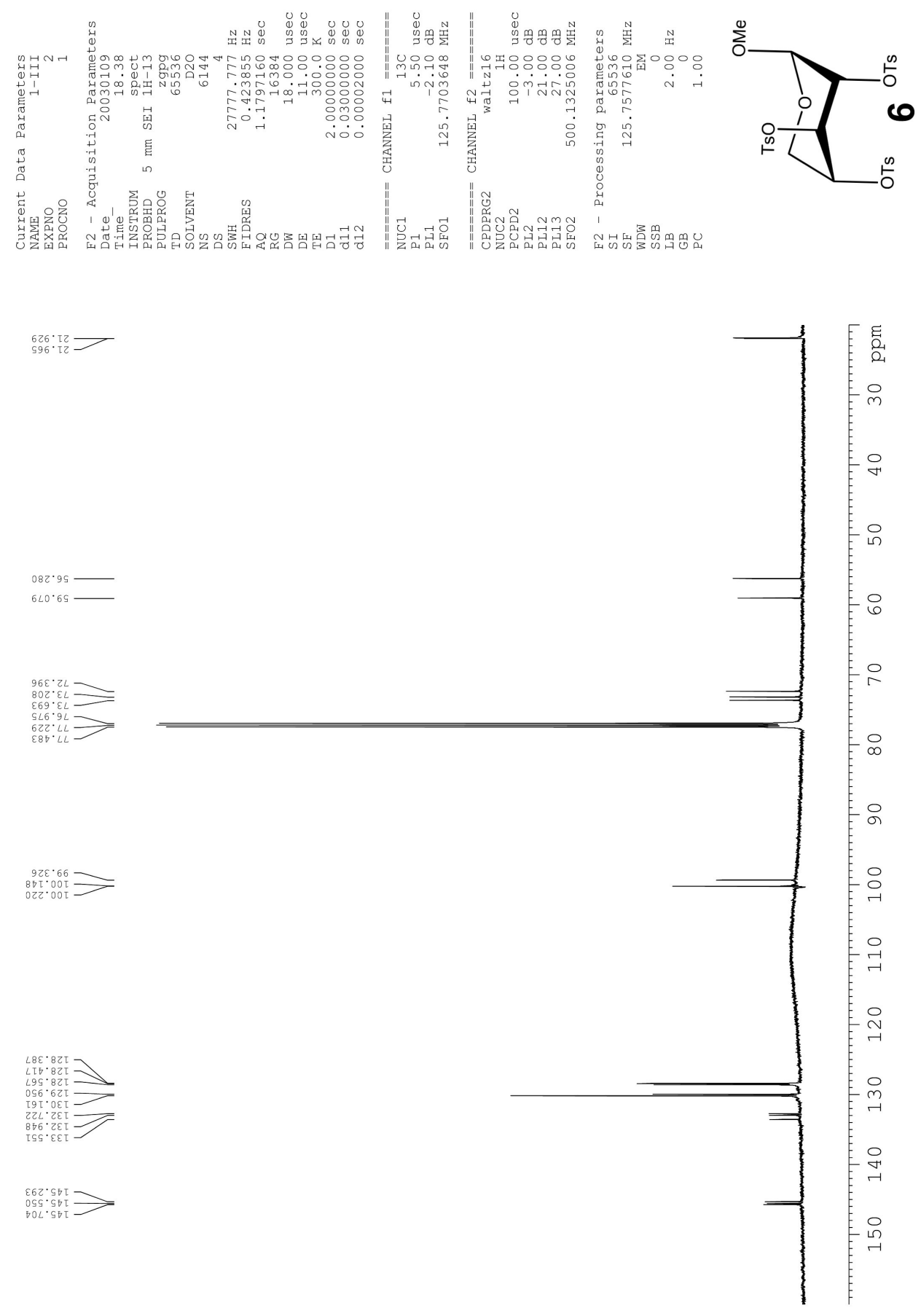


\section{Methyl 4- $O$-Benzoyl-2,3-bis-( $O$-4-toluenesulfonyl)- $\alpha$-L-arabinopyranoside (7).}

(1) From (6): The tritosylate (6) (250 mg, $0.399 \mathrm{mmol})$ and sodium benzoate (510 $\mathrm{mg}, 3.54 \mathrm{mmol})$ were suspended in dry DMF $(15 \mathrm{ml})$ and heated for $20 \mathrm{~h}$ at $130{ }^{\circ} \mathrm{C}$. The cooled mixture was evaporated, and then the residue was extracted with EtOAc $(50 \mathrm{ml})$. The filtered extract was washed with $5 \% \mathrm{HCl}(2 \times 50 \mathrm{ml})$, saturated $\mathrm{NaHCO}_{3}(2 \times 50 \mathrm{ml})$ and brine $(50 \mathrm{ml})$, then dried $\left(\mathrm{MgSO}_{4}\right)$ and evaporated to a crystalline solid. This was triturated with cold hexane to give the monobenzoate (7) as colorless crystals (140 mg, 61\%), m.p. $79-81{ }^{\circ} \mathrm{C} .[\alpha]_{\mathrm{D}}-1.4\left(c\right.$ 0.3, $\left.\mathrm{CHCl}_{3}\right)$. ES-MS: $577(\mathrm{MH})^{+}, 594\left(\mathrm{M}+\mathrm{NH}_{4}\right)^{+}, 599$ $(\mathrm{M}+\mathrm{Na})^{+} .{ }^{1} \mathrm{H}$ NMR $\left(500 \mathrm{MHz}, \mathrm{CDCl}_{3}\right) 2.25$ (s, 3H, Me); 2.42 (s, 3H, Me); 3.27 (s, 3H, OMe); 3.58 (ddd, 1H, $J=0.5,3.1,11.6 \mathrm{~Hz}, \mathrm{H} 5 \mathrm{a}) ; 4.08$ (dd, 1H, $J=7.0,11.2 \mathrm{~Hz}, \mathrm{H} 5 \mathrm{~b})$; $4.40(\mathrm{~d}, 1 \mathrm{H}, J=4.1 \mathrm{~Hz}, \mathrm{H} 1) ; 4.73(\mathrm{dd}, 1 \mathrm{H}, J=3.4,6.4 \mathrm{~Hz}, \mathrm{H} 3) ; 4.78(\mathrm{dd}, 1 \mathrm{H}, J=4.1,6.5$ $\mathrm{Hz}, \mathrm{H} 2$ ); 5.28 (dt, 1H, $J=3.4,6.9 \mathrm{~Hz}, \mathrm{H} 4) ; 7.03$ (br d, 2H, $J=11.2 \mathrm{~Hz}, \mathrm{Ar}-\mathrm{H}) ; 7.33$ (br $\mathrm{d}, 2 \mathrm{H}, J=9.3 \mathrm{~Hz}, \mathrm{Ar}-\mathrm{H}) ; 7.38(\mathrm{br} \mathrm{t}, 2 \mathrm{H}, J=7.9 \mathrm{~Hz}, \mathrm{Ar}-\mathrm{H}) ; 7.54(\mathrm{t}, 1 \mathrm{H}, J=7.5 \mathrm{~Hz}, \mathrm{Ar}-$ $\mathrm{H}) ; 7.57(\mathrm{~d}, 2 \mathrm{H}, J=8.3, \mathrm{Ar}-\mathrm{H}) ; 7.80-7.84(\mathrm{~m}, 2 \mathrm{H}, \mathrm{Ar}-\mathrm{H}) .{ }^{13} \mathrm{C} \mathrm{NMR}(125 \mathrm{MHz}, \mathrm{CDCl})$ $21.7,21.8,56.5,60.4,66.8,74.5,75.4,100.1,128.3,128.4,128.5,128.6,129.1,129.2$, 129.9, 132.8, 133.5, 133.6, 145.1, 145.2. 165.0. HRMS (TOF) calcd for $\mathrm{C}_{27} \mathrm{H}_{29} \mathrm{O}_{10} \mathrm{~S}_{2}$ $(\mathrm{M}+\mathrm{H})^{+}$577.1202, found 577.1208.

(2) From (9): A solution of the ditosylate $(9)(7.0 \mathrm{mg}, 0.015 \mathrm{mmol})$ in pyridine (3 $\mathrm{ml})$ was treated with benzoyl chloride $(0.15 \mathrm{ml}, 0.18 \mathrm{~g}, 1.3 \mathrm{mmol})$. After stirring overnight at room temperature, the solution was evaporated and the residue was dissolved in EtOAc $(10 \mathrm{ml})$. This solution was washed with $5 \% \mathrm{HCl}(3 \times 10 \mathrm{ml})$, saturated $\mathrm{NaHCO}_{3}$ 
(2x10ml) and brine $(10 \mathrm{ml})$, then dried $\left(\mathrm{MgSO}_{4}\right)$ and evaporated to give the benzoate (7)

( $8.5 \mathrm{mg}, 100 \%$ ), identical by NMR and TLC to the sample prepared from (6) (above). 

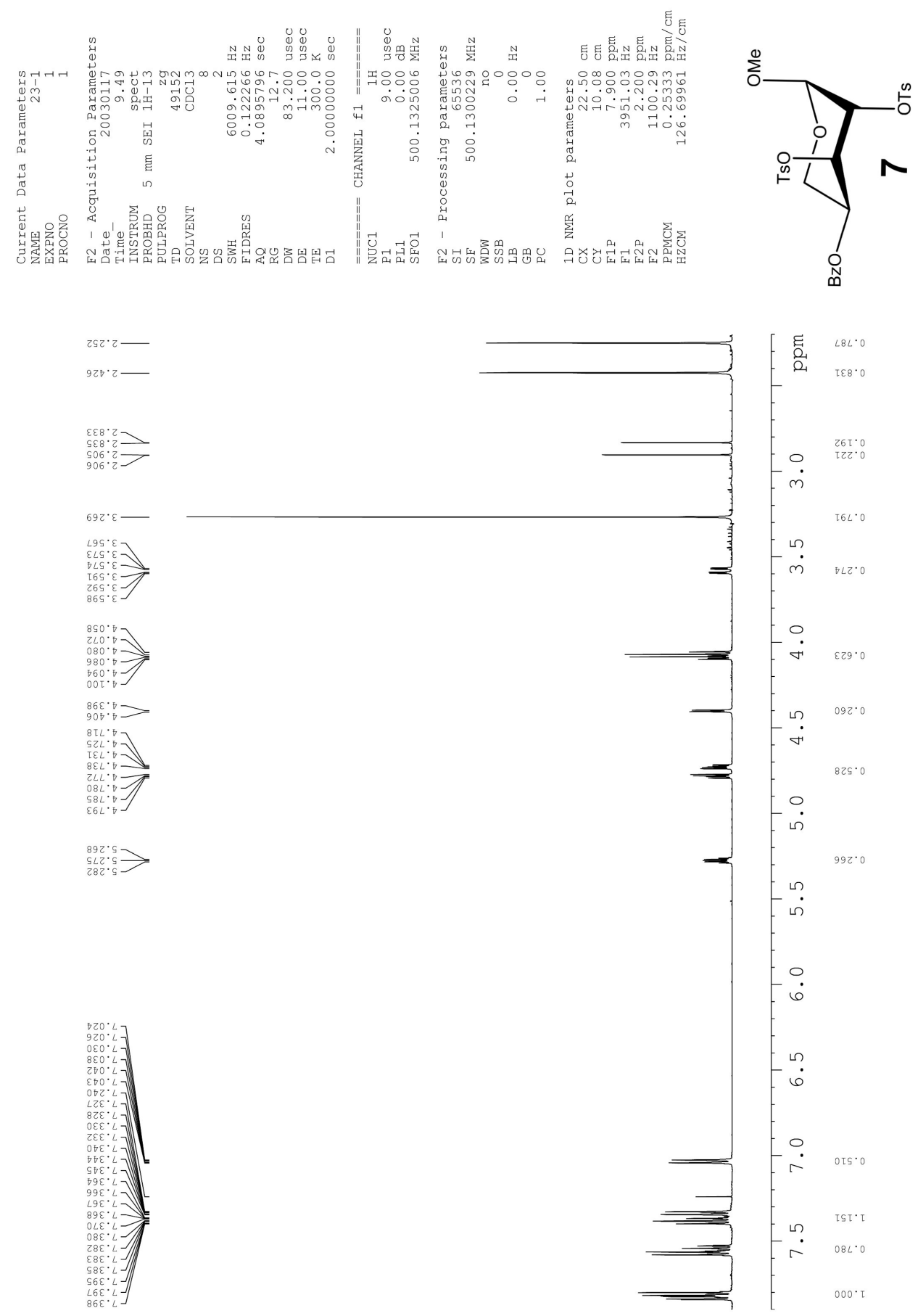

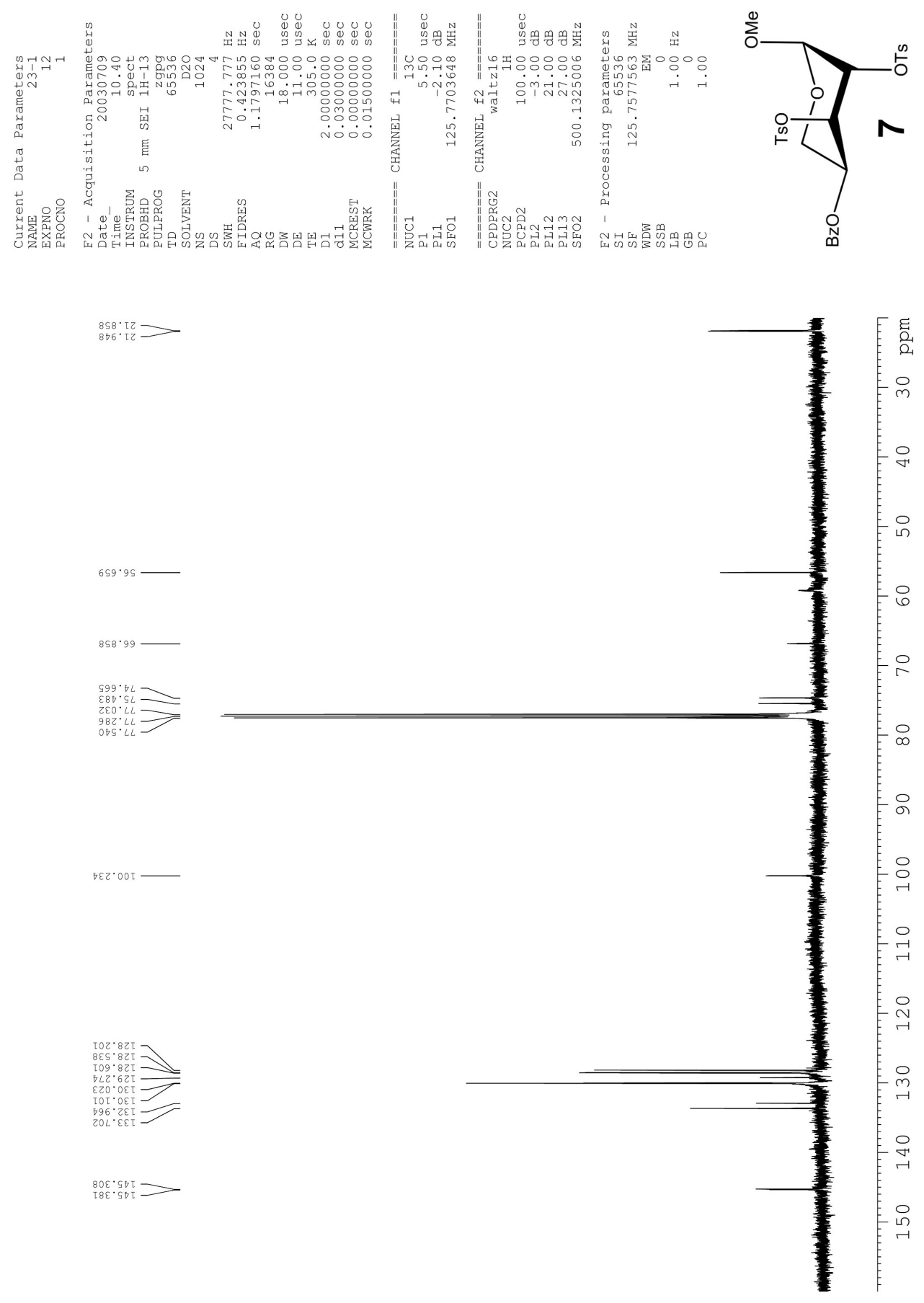


\section{Methyl 4-Azido-2,3-Bis-(O-4-toluenesulfonyl)-4-deoxy- $\alpha$-L-arabino pyranoside (8).}

The tritosylate (6) $(50 \mathrm{mg}, 80 \mu \mathrm{mol})$ and sodium azide $(27 \mathrm{mg}, 0.41 \mathrm{mmol})$ were suspended in dry DMF $(5 \mathrm{ml})$ and heated for $20 \mathrm{~h}$ at $130{ }^{\circ} \mathrm{C}$. The cooled mixture was evaporated, and then the residue was extracted with EtOAc $(50 \mathrm{ml})$. The filtered extract was washed with $5 \% \mathrm{HCl}(2 \times 50 \mathrm{ml})$, saturated $\mathrm{NaHCO}_{3}(2 \times 50 \mathrm{ml})$ and brine $(50 \mathrm{ml})$, then dried $\left(\mathrm{MgSO}_{4}\right)$ and evaporated to a yellow crystalline solid. This was recrystallized from EtOAc/hexane to give the azido derivative (8) as colorless crystals (45 $\mathrm{mg}, 98 \%$ ), m.p. $176-178{ }^{\circ} \mathrm{C}\left(\right.$ softening at $\left.165{ }^{\circ} \mathrm{C}\right) .[\alpha]_{\mathrm{D}}-11.8\left(c 0.2, \mathrm{CHCl}_{3}\right)$. ES-MS: $498(\mathrm{MH})^{+}$, $515\left(\mathrm{M}+\mathrm{NH}_{4}\right)^{+}, 520(\mathrm{M}+\mathrm{Na})^{+} .{ }^{1} \mathrm{H} \mathrm{NMR}(500 \mathrm{MHz}, \mathrm{CDCl}) 2.42(\mathrm{~s}, 3 \mathrm{H}, \mathrm{Me}) ; 2.45(\mathrm{~s}$, 3H, Me); 3.17 (s, 3H, OMe); 3.53 (dd, 1H, $J=2.7,11.7 \mathrm{~Hz}, \mathrm{H} 5 \mathrm{e}) ; 3.93(\mathrm{dd}, 1 \mathrm{H}, J=6.3$, $11.6 \mathrm{~Hz}, \mathrm{H} 5 \mathrm{a}) ; 3.96-3.99$ (m, 1H, H4); 4.26 (d, 1H, $J=4.4 \mathrm{~Hz}, \mathrm{H1}$ ); 4.64 (dd, 1H, $J=$ 3.1, $6.7 \mathrm{~Hz}, \mathrm{H} 3$ ); 4.66 (dd, 1H, $J=4.3,6.8 \mathrm{~Hz}, \mathrm{H} 2) ; 7.32-7.36$ (m, 4H, Ar-H); 7.78 (br d, $2 \mathrm{H}, J 8.6 \mathrm{~Hz}, \mathrm{Ar}-\mathrm{H}) ; 7.81(\mathrm{br} \mathrm{d}, 2 \mathrm{H}, J=8.5 \mathrm{~Hz}, \mathrm{Ar}-\mathrm{H}) .{ }^{13} \mathrm{C} \mathrm{NMR}\left(125 \mathrm{MHz}, \mathrm{CDCl}_{3}\right)$ $22.1,22.2,56.8,57.1,60.3,75.5,75.7,100.1,128.7,128.9,130.2,130.4,132.7,133.9$, 145.6, 146.1. HRMS (TOF) calcd for $\mathrm{C}_{20} \mathrm{H}_{24} \mathrm{~N}_{3} \mathrm{O}_{8} \mathrm{~S}_{2}(\mathrm{M}+\mathrm{H})^{+}$498.1005, found 498.1002. 


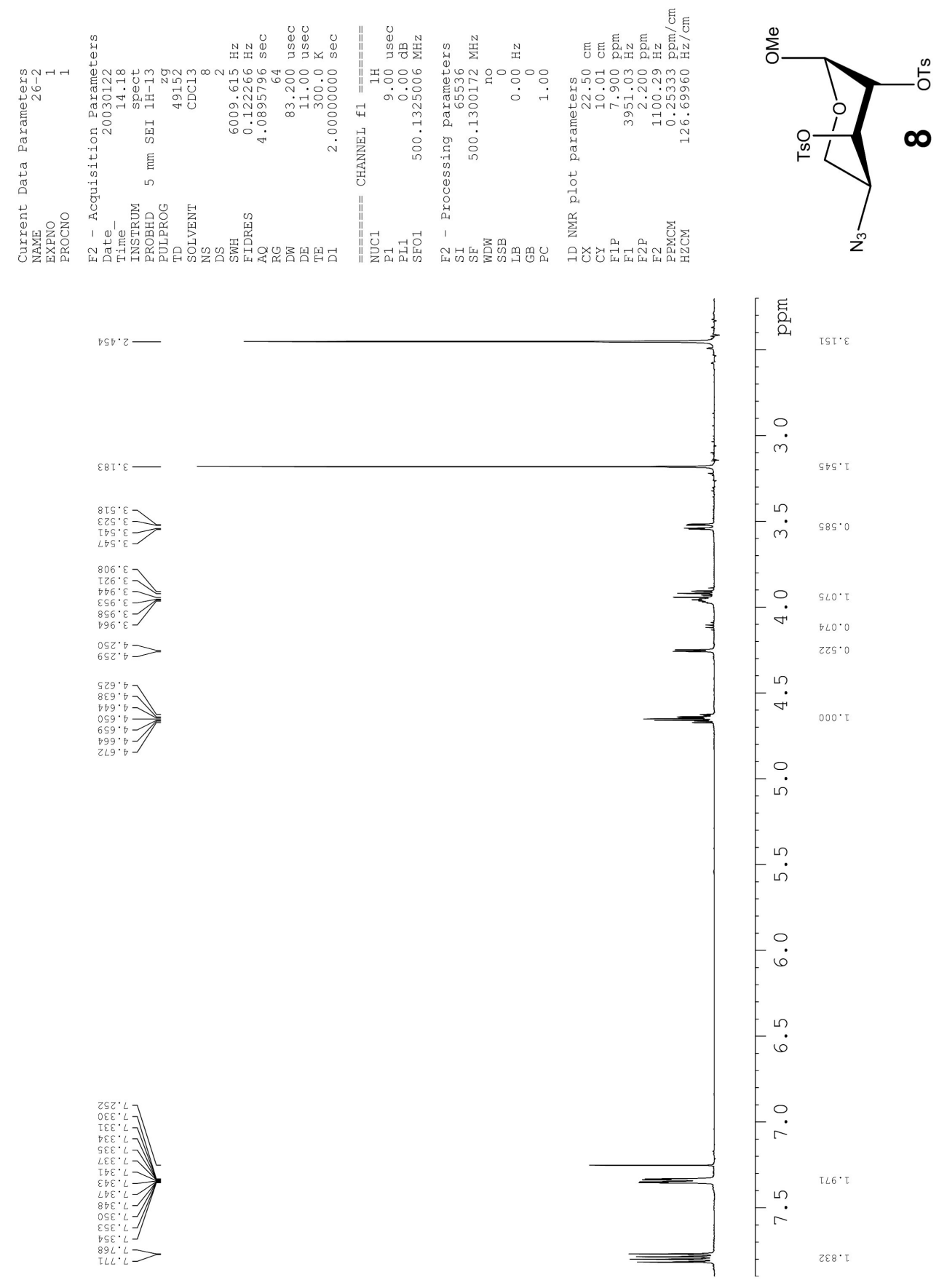



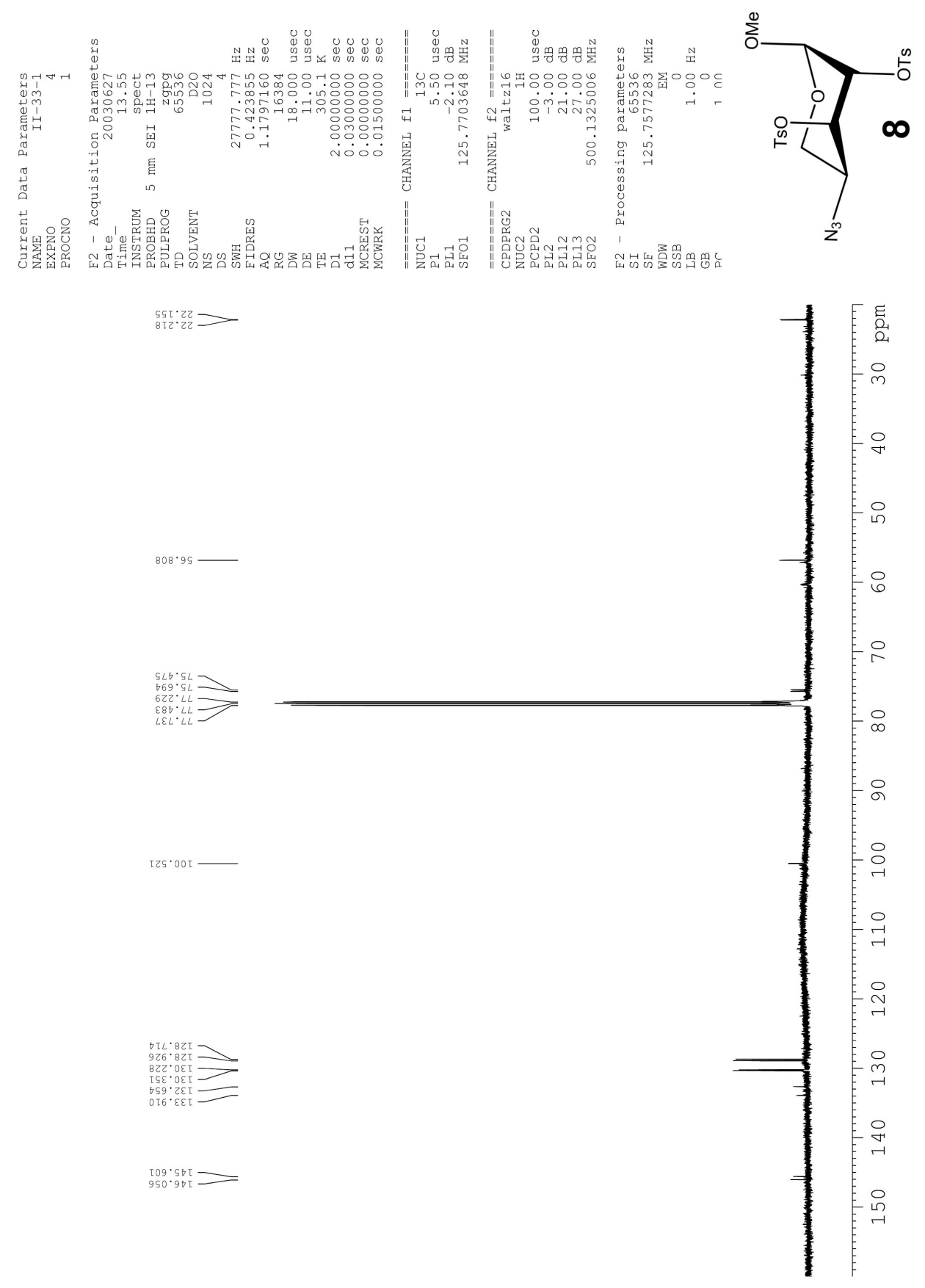
Methyl 2,3-Bis-(O-4-toluenesulfonyl)- $\alpha$-L-arabinopyranoside (9). The tritosylate (6) (100 mg, $0.16 \mathrm{mmol})$ and sodium nitrite $(100 \mathrm{mg}, 1.45 \mathrm{mmol})$ were suspended in dry DMF (5 ml) and heated at $130{ }^{\circ} \mathrm{C}$ for $72 \mathrm{~h}$. The cooled mixture was evaporated, and the residue was extracted with EtOAc $(20 \mathrm{ml})$. The organic extract was washed with 5\% $\mathrm{HCl}$ $(2 \times 20 \mathrm{ml})$, then saturated $\mathrm{NaHCO}_{3}(2 \times 20 \mathrm{ml})$ and brine $(15 \mathrm{ml})$, then dried $\left(\mathrm{MgSO}_{4}\right)$ and evaporated to a colorless foam. This was purified by column chromatography (eluent: EtOAc/ $\mathrm{CHCl} /$ hexane 3:2:1) to give the ditosylate (9) as colorless crystals (42 mg, 56\%), m.p. $122-123{ }^{\circ} \mathrm{C} .[\alpha]_{\mathrm{D}}-2.9\left(c\right.$ 0.5, $\left.\mathrm{CHCl}_{3}\right)$. ES-MS: $473(\mathrm{MH})^{+}, 490\left(\mathrm{M}+\mathrm{NH}_{4}\right)^{+}, 495$ $(\mathrm{M}+\mathrm{Na})^{+} . R_{f}\left(3: 2: 1 \mathrm{EtOAc} / \mathrm{CHCl}_{3} / \mathrm{hexane}\right): 0.18 .{ }^{1} \mathrm{H} \mathrm{NMR}(500 \mathrm{MHz}, \mathrm{CDCl}) 2.43(\mathrm{~s}$, 3H, Me); 2.44 (s, 3H, Me); 3.14 (s, 3H, OMe); 3.49 (dd, 1H, $J=2.6,12.5 \mathrm{~Hz}, \mathrm{H} 5 \mathrm{a}$ ); 3.89 (dd, 1H, $J=5.1,12.4 \mathrm{~Hz}, \mathrm{H} 5 \mathrm{~b}) ; 4.13-4.18(\mathrm{~m}, 1 \mathrm{H}, \mathrm{H} 4) ; 4.19(\mathrm{~d}, 1 \mathrm{H}, J=5.3 \mathrm{~Hz}, \mathrm{H} 1$ ); $4.57(\mathrm{dd}, 1 \mathrm{H}, J=3.3,7.5 \mathrm{~Hz}, \mathrm{H} 3) ; 4.67(\mathrm{dd}, 1 \mathrm{H}, J=5.3,7.4 \mathrm{~Hz}, \mathrm{H} 2) ; 7.30(\mathrm{br} \mathrm{d}, 2 \mathrm{H}, J=$ $8.0 \mathrm{~Hz}, \mathrm{Ar}-\mathrm{H}) ; 7.34$ (br d, 2H, $J=8.0 \mathrm{~Hz}, \mathrm{Ar}-\mathrm{H}) ; 7.72($ br d, $2 \mathrm{H}, J=8.3 \mathrm{~Hz}, \mathrm{Ar}-\mathrm{H}) ; 7.80$ (br d, $2 \mathrm{H}, J=8.2, \mathrm{Ar}-\mathrm{H})$; $\mathrm{OH}$ not observed. ${ }^{13} \mathrm{C}$ NMR $\left(125 \mathrm{MHz}, \mathrm{CDCl}_{3}\right)$ 22.1, 22.2, $56.8,63.2,66.5,76.4,78.3,101.0,128.7,128.8,130.1,130.4,133.0,134.7,145.3,146.0$. HRMS (TOF) calcd for $\mathrm{C}_{20} \mathrm{H}_{25} \mathrm{O}_{9} \mathrm{~S}_{2}(\mathrm{M}+\mathrm{H})^{+} 473.0940$, found 473.0950 . 


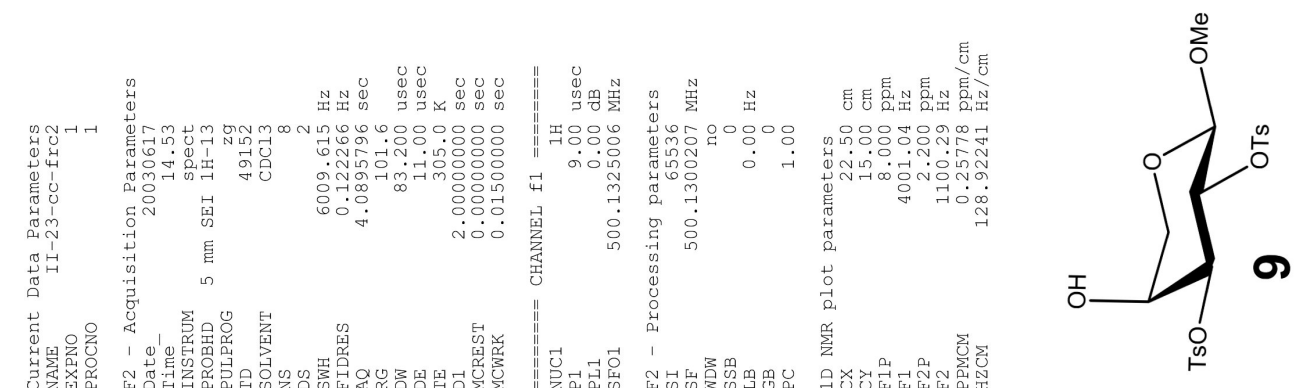

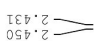
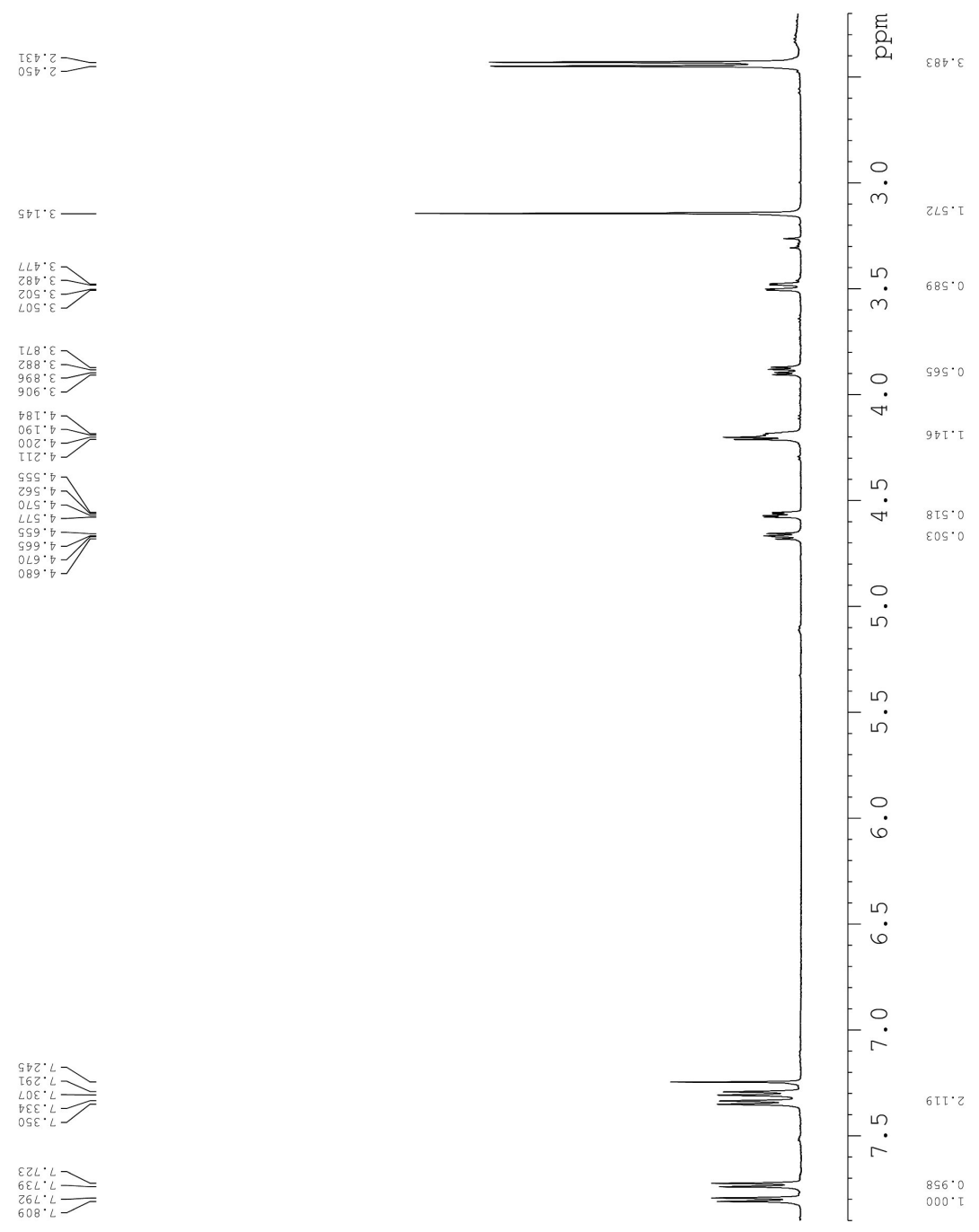

$E 2 L: L$
$6 \varepsilon L$
$26 L: L$
$608 . L$ 


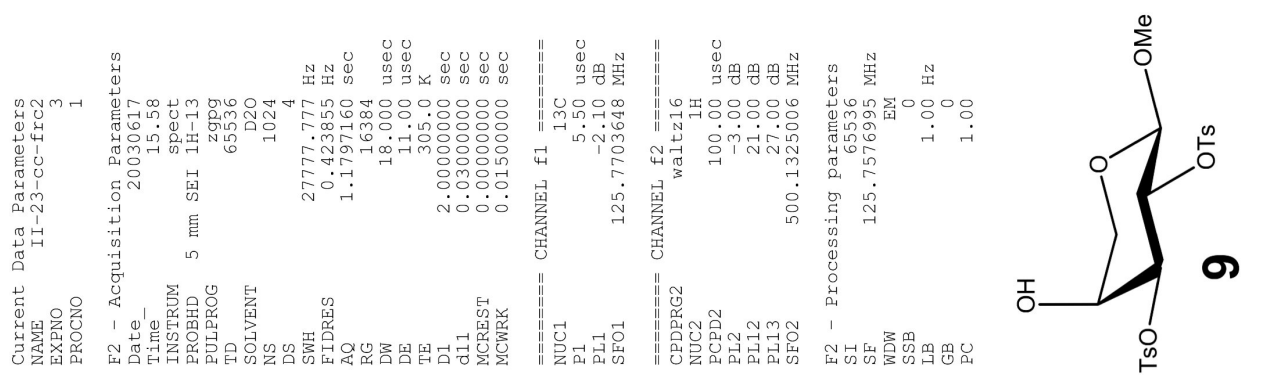

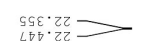

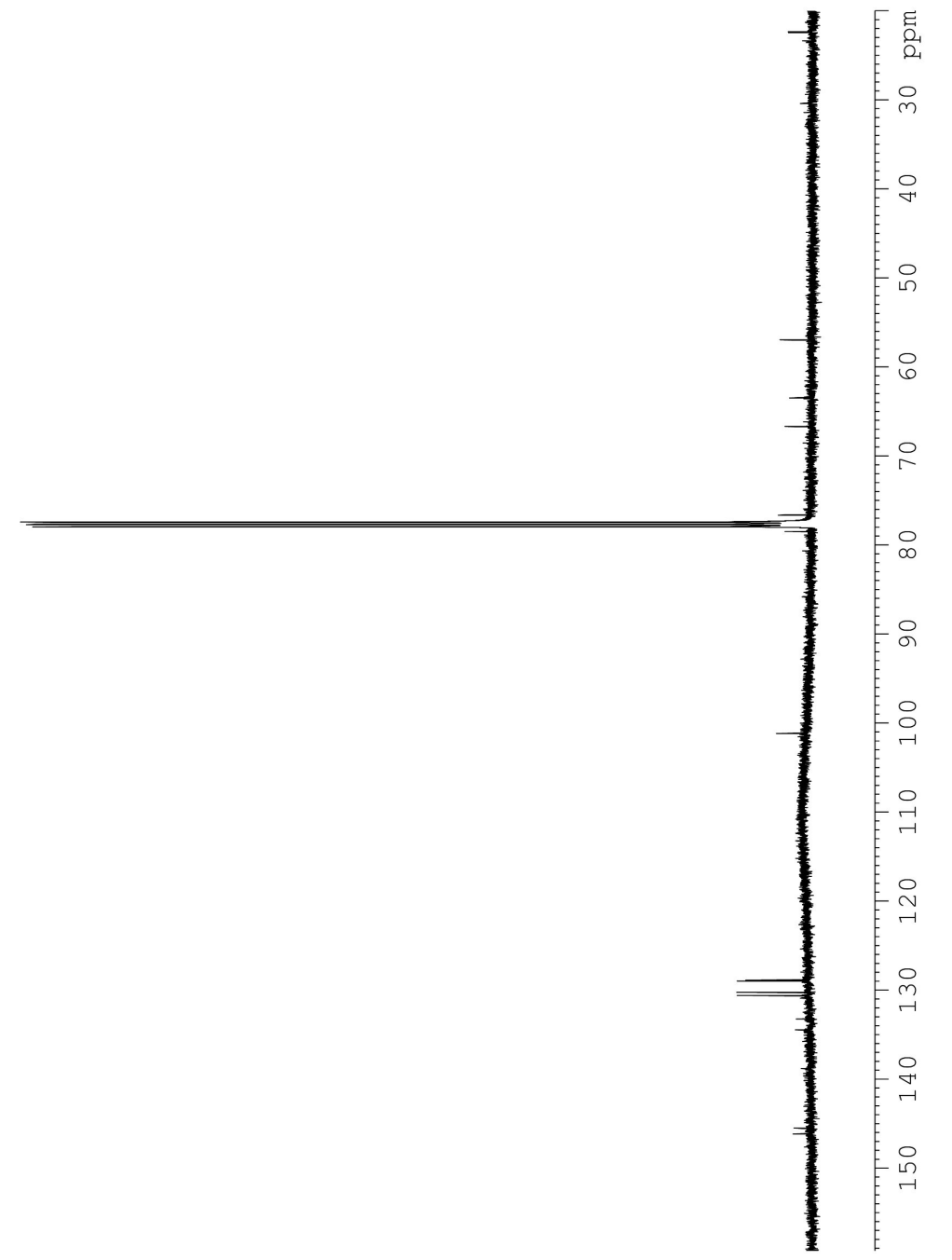

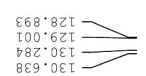

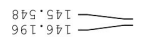

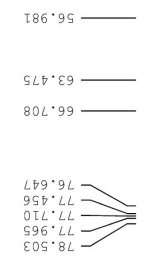

8LT.TOT 
Methyl 2,3,4-Tris-( $O$-methane sulfonyl)- $\beta$-D-xylopyranoside (10). Methyl $\beta$-Dxylopyranoside (5) $(500 \mathrm{mg}, 3.05 \mathrm{mmol})$ was added to a stirred, chilled $\left(0{ }^{\circ} \mathrm{C}\right)$ solution of methanesulfonyl chloride $(0.95 \mathrm{ml}, 1.40 \mathrm{~g}, 12.2 \mathrm{mmol})$ in pyridine $(10 \mathrm{ml})$. After standing for $7 \mathrm{~d}$, the dark crystalline mass was poured onto ice $(50 \mathrm{ml})$. After the ice had melted, the dark mixture was shaken with $\mathrm{CH}_{2} \mathrm{Cl}_{2}(3 \times 50 \mathrm{ml})$ and filtered through a layer of Celite. The organic phase was washed with $5 \% \mathrm{HCl}(3 \times 50 \mathrm{ml})$, saturated $\mathrm{NaHCO}_{3}$ $(100 \mathrm{ml})$ and brine $(50 \mathrm{ml})$, then dried $\left(\mathrm{MgSO}_{4}\right)$ and evaporated to a yellow foam. The product was dissolved in a little methanol and slow evaporation of the solvent yielded tan crystals of the trimesylate (10) $\left(1.19\right.$ g, 98\%), m.p. $137.5-138.5^{\circ} \mathrm{C}$ (lit. ${ }^{5}$ m.p. 147.5148.5). $[\alpha]_{\mathrm{D}}-33.0\left(c 0.3, \mathrm{CHCl}_{3}\right)\left(\right.$ lit. $^{5}[\alpha]_{\mathrm{D}}-33.6\left(c 1.4, \mathrm{CHCl}_{3}\right) . R_{f}(\mathrm{EtOAc}): 0.57 .{ }^{1} \mathrm{H}$ NMR (500 MHz, CDCb) 3.15 (s, 3H, Me); 3.16 (s, 3H, Me); 3.22 (s, 3H, Me); 3.49 (dd, $1 \mathrm{H}, J=10.1,12.0 \mathrm{~Hz}, \mathrm{H} 5 \mathrm{a}) ; 3.55$ (s, 3H, OMe); $4.32(\mathrm{dd}, 1 \mathrm{H}, J=5.7,12.0 \mathrm{~Hz}, \mathrm{H} 5 \mathrm{~b})$; $4.40(\mathrm{~d}, 1 \mathrm{H}, J=7.6 \mathrm{~Hz}, \mathrm{H1}) ; 4.49$ (dd, 1H, $J=7.6,9.4 \mathrm{~Hz}, \mathrm{H} 2) ; 4.65$ (ddd, $1 \mathrm{H}, J=5.7$, 9.1, $10.1 \mathrm{~Hz}, \mathrm{H} 4) ; 4.85$ (t, $1 \mathrm{H}, J=9.3, \mathrm{H} 3) .{ }^{13} \mathrm{C} \mathrm{NMR}(125 \mathrm{MHz}, \mathrm{CDCl}$ ) 38.4, 39.3, 39.6, 57.1, 63.3, 74.0, 77.6, 78.3, 101.4.

Methyl $\beta$-L-Arabinopyranoside (12). A mixture of L-arabinose (11) (11.0 g, 73.3 mmol) and ion-exchange resin (Amberlite ${ }^{\circledR}$ IRA-120, $20.0 \mathrm{~g}$ ) in anhydrous methanol $(150 \mathrm{ml})$ was heated under reflux overnight. The cooled mixture was filtered and evaporated to a cream solid. This was recrystallized from ethanol to give the pure $\beta$ anomer (12) as colorless crystals $\left(4.40\right.$ g, 37\%), m.p. $166.5-168.5^{\circ} \mathrm{C}$ (lit. ${ }^{6}$ m.p. 167-169 $\left.{ }^{\circ} \mathrm{C}\right) .[\alpha]_{\mathrm{D}} 240\left(c 0.6, \mathrm{H}_{2} \mathrm{O}\right)\left(\right.$ lit. $\left.\left.^{7}[\alpha]_{\mathrm{D}} 243\left(c 0.6, \mathrm{H}_{2} \mathrm{O}\right)\right) .{ }^{1} \mathrm{H} \mathrm{NMR}\left(500 \mathrm{MHz},\left(\mathrm{CD}_{3}\right)_{2} \mathrm{SO}\right)\right)$ 3.30 (s, 3H, OMe); 3.44 (dd, 1H, $J=2.9,11.9 \mathrm{~Hz}, \mathrm{H} 5) ; 3.52-3.62$ (m, 3H, H2,3,5); 3.69 
(m, 1H, H4); $4.45(\mathrm{~d}, 1 \mathrm{H}, J=3.5 \mathrm{~Hz}, \mathrm{H1}) ; 4.49-4.54(\mathrm{~m}, 3 \mathrm{H}, 3 \mathrm{xOH}) .{ }^{13} \mathrm{C} \mathrm{NMR}(125$ $\left.\left.\mathrm{MHz},\left(\mathrm{CD}_{3}\right)_{2} \mathrm{SO}\right)\right)$ 54.8, 62.9, 68.2, 68.6, 69.1, 100.6.

Methyl 2,3,4-Tris-( $O$-4-toluenesulfonyl)- $\beta$-L-arabinopyranoside (13). Methyl $\beta$-Larabinopyranoside (12) $(1.33 \mathrm{~g}, 8.10 \mathrm{mmol})$ was added portionwise to a chilled $\left(0{ }^{\circ} \mathrm{C}\right)$ solution of 4-toluenesulfonyl chloride $(6.18 \mathrm{~g}, 32.4 \mathrm{mmol})$ in pyridine $(20 \mathrm{ml})$. After standing for 7 days at room temperature, the solution was poured into $100 \mathrm{ml}$ of ice water, and the product separated as a sticky mass. The mixture was extracted with $\mathrm{CHCl}_{3},(3 \times 100 \mathrm{ml})$ and the combined extracts were washed with $5 \% \mathrm{HCl}(2 \times 50 \mathrm{ml})$, water $(50 \mathrm{ml})$ and brine $(50 \mathrm{ml})$, then dried $\left(\mathrm{MgSO}_{4}\right)$ and evaporated to a colorless foam $(4.95 \mathrm{~g})$. This was recrystallized twice from ethanol to give the tritosylate (13) as colorless crystals $(3.46$ g, $68 \%)$, m.p. $112-113{ }^{\circ} \mathrm{C}$ (lit. ${ }^{3}$ m.p. $\left.116-117{ }^{\circ} \mathrm{C}\right) .[\alpha]_{\mathrm{D}} 97.7(c$ 0.9, $\left.\mathrm{CHCl}_{3}\right)\left(\right.$ lit. $\left.^{8}[\alpha]_{\mathrm{D}} 101\left(c 0.9, \mathrm{CHCl}_{3}\right)\right)$. ES-MS: $627(\mathrm{MH})^{+}, 644\left(\mathrm{M}+\mathrm{NH}_{4}\right)^{+}, 649$ $(\mathrm{M}+\mathrm{Na})^{+} .{ }^{1} \mathrm{H}$ NMR (500 MHz, CDCl) $): 2.43$ (s, 3H, Me); 2.44 (s, 3H, Me); 2.46 (s, 3H, Me); 3.25 (s, 3H, OMe); 3.75 (dd, 1H, $J=0.7,13.3 \mathrm{~Hz}, \mathrm{H} 5 \mathrm{a}) ; 3.85$ (dd, $1 \mathrm{H}, J=2.3,13.3$ Hz, H5b); $4.64(\mathrm{dd}, 1 \mathrm{H}, J=3.4,10.2 \mathrm{~Hz}, \mathrm{H} 2) ; 4.75(\mathrm{dd}, 1 \mathrm{H}, J=3.3,10.1 \mathrm{~Hz}, \mathrm{H} 3) ; 4.77$ $(\mathrm{d}, 1 \mathrm{H}, J=3.4 \mathrm{~Hz}, \mathrm{H1}) ; 4.98(\mathrm{~m}, 1 \mathrm{H}, \mathrm{H} 4) ; 7.26(\mathrm{br} \mathrm{d}, 2 \mathrm{H}, J=8.2 \mathrm{~Hz}, \mathrm{Ar}-\mathrm{H}) ; 7.28$ (br d, $2 \mathrm{H}, J=8.3 \mathrm{~Hz}, \mathrm{Ar}-\mathrm{H}) ; 7.34(\mathrm{brd}, 2 \mathrm{H}, J=8.3 \mathrm{~Hz}, \mathrm{Ar}-\mathrm{H}) ; 7.62(\mathrm{br} \mathrm{d}, 2 \mathrm{H}, J=8.3 \mathrm{~Hz}, \mathrm{Ar}-$ $\mathrm{H}) ; 7.64$ (br d, $2 \mathrm{H}, J=8.3 \mathrm{~Hz}, \mathrm{Ar}-\mathrm{H}) ; 7.78$ (br d, $2 \mathrm{H}, J=8.2 \mathrm{~Hz}, \mathrm{Ar}-\mathrm{H}) .{ }^{13} \mathrm{C}$ NMR $(125$ $\left.\mathrm{Hz}, \mathrm{CDCl}_{3}\right) 21.7,21.8,21.8,56.0,60.3,72.1,73.3,97.9,128.1,128.2,128.3,129.8$, $129.9,130.1,132.5,133.2,133.3,145.1,145.2,145.3$. 

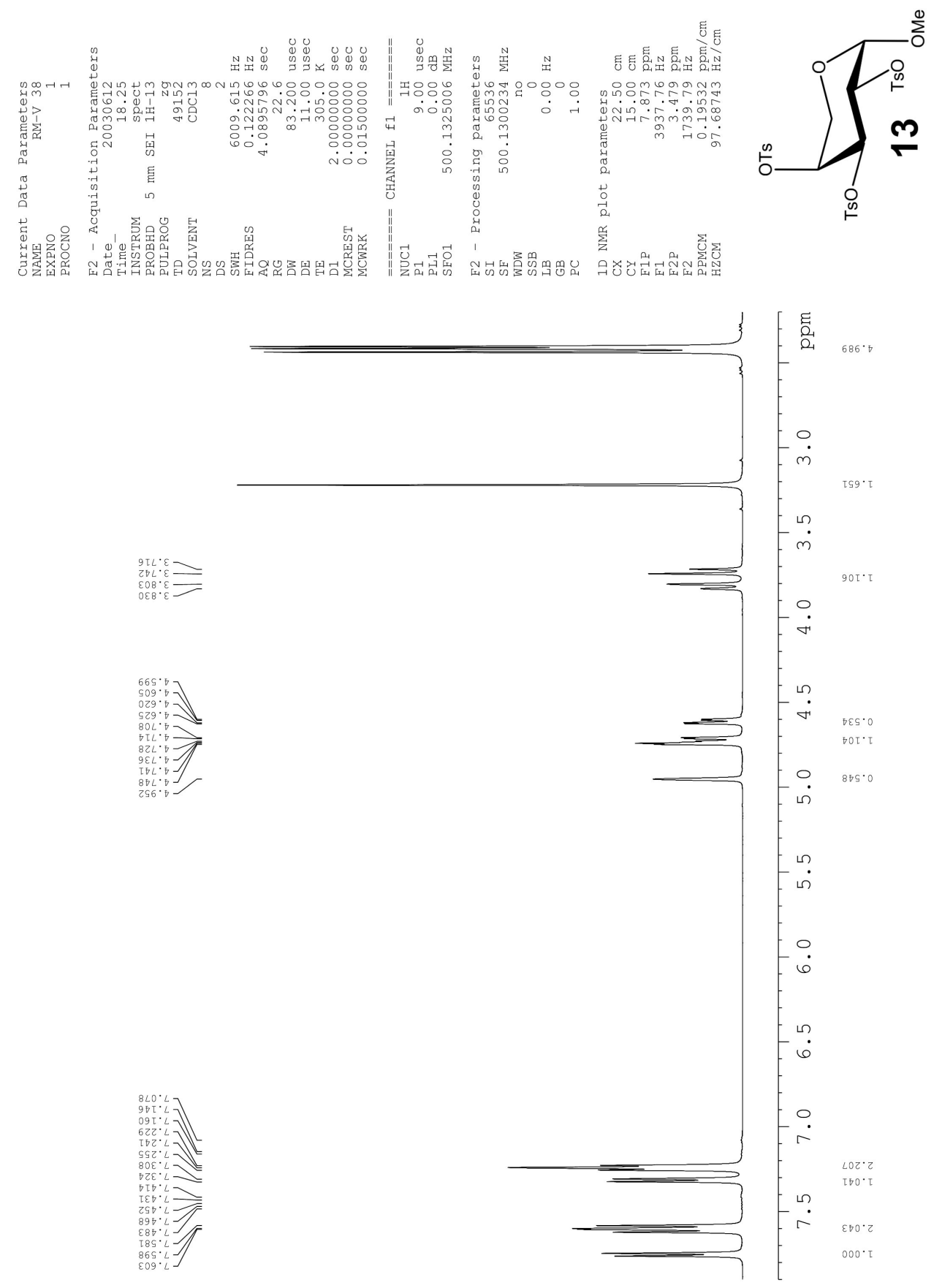

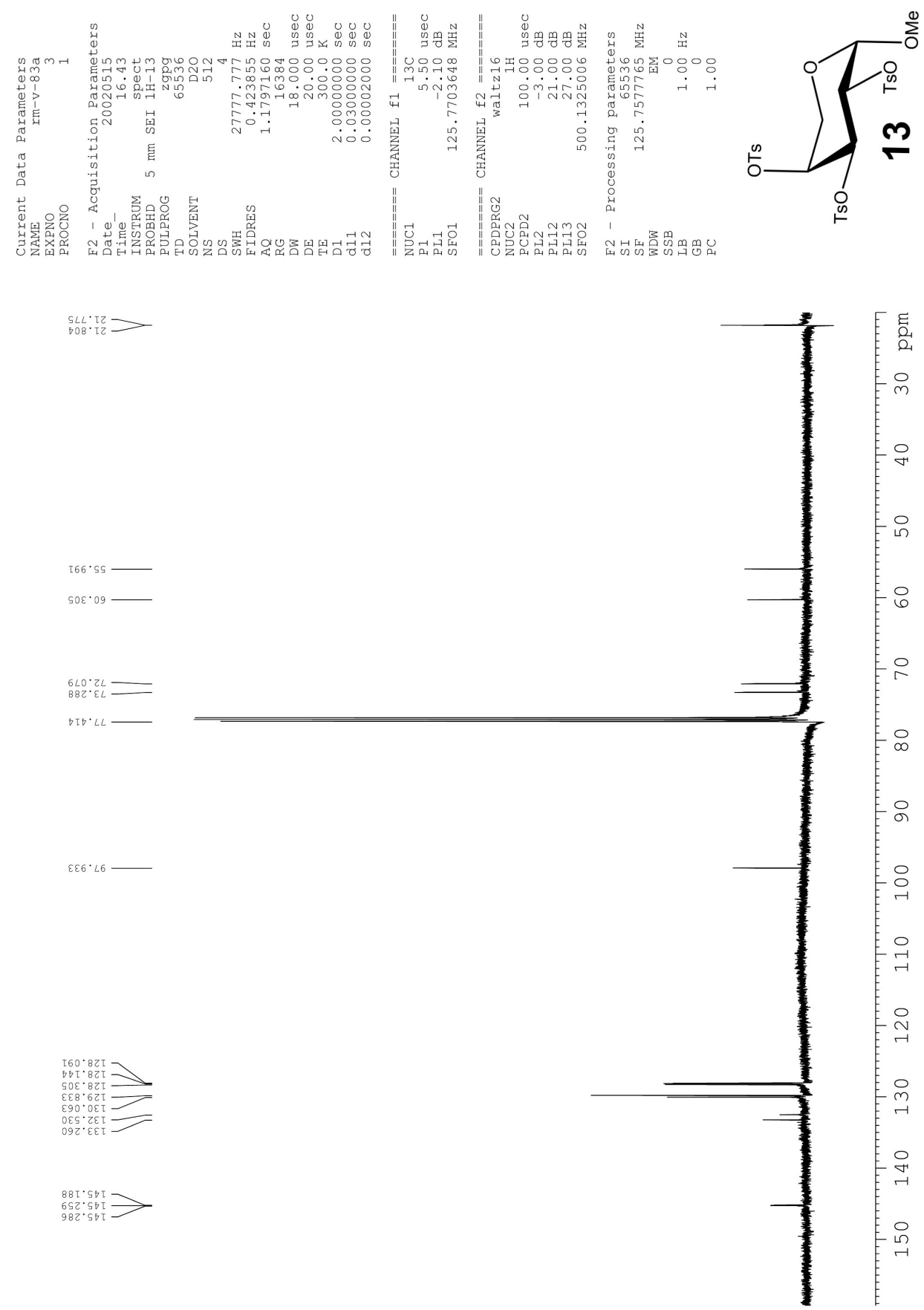

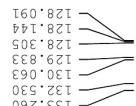

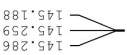

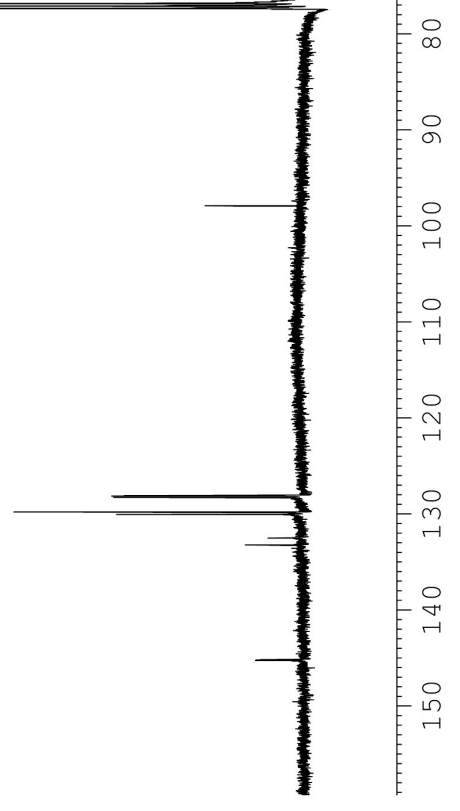




\section{Methyl 4- $O$-Benzoyl-2,3-bis-(O-4-toluenesulfonyl)- $\alpha$-D-xylopyranoside (14).}

(1) From (13): A suspension of the arabinose tritosylate (13) (200 mg, $0.32 \mathrm{mmol})$ and sodium benzoate $(460 \mathrm{mg}, 3.19 \mathrm{mmol})$ in dry DMF $(25 \mathrm{ml})$ was heated at $130{ }^{\circ} \mathrm{C}$ for 4 days. The cooled reaction mixture was evaporated and the residue was extracted with EtOAc $(45 \mathrm{ml})$. The organic extract was washed with $5 \% \mathrm{HCl}(2 \times 25 \mathrm{ml})$, saturated $\mathrm{NaHCO}_{3}(2 \times 25 \mathrm{ml})$ and brine $(10 \mathrm{ml})$, then dried $\left(\mathrm{MgSO}_{4}\right)$ and evaporated to a foam. This was triturated with hexane to give the monobenzoate (14) as a gum (120 $\mathrm{mg}, 65 \%)$. Crystallization from EtOAc/hexane gave colorless crystals $(73 \mathrm{mg}, 40 \%)$, m.p. $58-60{ }^{\circ} \mathrm{C}$. $R_{f}\left(1: 2: 3 \mathrm{EtOAc} / \mathrm{CHCl}_{3} /\right.$ hexane $): 0.55 .[\alpha]_{\mathrm{D}}-26.0\left(c 0.4, \mathrm{CHCl}_{3}\right)\left(\right.$ lit. $^{4}[\alpha]_{\mathrm{D}}-24.1(c$ 4.6, $\left.\mathrm{CHCl}_{3}\right) . \mathrm{ES}-\mathrm{MS}: 577(\mathrm{MH})^{+}, 594\left(\mathrm{M}+\mathrm{NH}_{4}\right)^{+}, 599(\mathrm{M}+\mathrm{Na})^{+} .{ }^{1} \mathrm{H} \mathrm{NMR}(500 \mathrm{MHz}$, $\left.\mathrm{CDCl}_{3}\right): 2.26$ (s, 3H, Me); 2.43 (s, 3H, Me); 3.37 (s, 3H, OMe); 3.63 (t, 1H, J=10.8 Hz, H5a); $3.88(\mathrm{dd}, 1 \mathrm{H}, J=6.1,11.0 \mathrm{~Hz}, \mathrm{H} 5 \mathrm{e}) ; 4.34(\mathrm{dd}, 1 \mathrm{H}, J=3.5,9.7 \mathrm{~Hz}, \mathrm{H} 2) ; 4.94$ (d, $1 \mathrm{H}, J=3.5 \mathrm{~Hz}, \mathrm{H} 1) ; 5.07-5.19(\mathrm{~m}, 1 \mathrm{H}, \mathrm{H} 4) ; 5.33$ (t, 1H, $J=9.5 \mathrm{~Hz}, \mathrm{H} 3) ; 7.09$ (br d, 2H, $J 8.6 \mathrm{~Hz}, \mathrm{Ar}-\mathrm{H}) ; 7.27$ (br d, 2H, $J=8.6 \mathrm{~Hz}, \mathrm{Ar}-\mathrm{H}) ; 7.41$ (br t, $2 \mathrm{H}, J=8.5 \mathrm{~Hz}, \mathrm{Ar}-\mathrm{H}) ; 7.56$ (br t, $1 \mathrm{H}, J=8.7 \mathrm{~Hz}, \mathrm{Ar}-\mathrm{H}) ; 7.64$ (br d, $4 \mathrm{H}, J=8.3 \mathrm{~Hz}, \mathrm{Ar}-\mathrm{H}) ; 7.97$ (br d, 2H, $J=8.4 \mathrm{~Hz}$, Ar-H). ${ }^{13} \mathrm{C}$ NMR $\left(125 \mathrm{~Hz}, \mathrm{CDCl}_{3}\right)$ 21.7, 21.8, 56.0, 58.4, 69.5, 75.8, 76.6, 97.6, 127.8, $128.4,128.9,129.7,129.8,130.1,130.2,132.6,133.5,134.2,144.6,145.4$. HRMS (TOF) calcd for $\mathrm{C}_{27} \mathrm{H}_{29} \mathrm{O}_{10} \mathrm{~S}_{2}(\mathrm{M}+\mathrm{H})^{+}$577.1202, found 577.1242.

(2) From (16): A solution of the alcohol (16) (10.9 $\mathrm{mg}, 0.023 \mathrm{mmol})$ in pyridine (3 $\mathrm{ml})$ was treated with benzoyl chloride $(0.15 \mathrm{ml}, 0.18 \mathrm{~g}, 1.3 \mathrm{mmol})$. After stirring overnight at room temperature, the solution was evaporated and the residue was dissolved in EtOAc $(15 \mathrm{ml})$. This solution was washed with $5 \% \mathrm{HCl}(2 \times 10 \mathrm{ml})$, saturated $\mathrm{NaHCO}_{3}$ 
(2x10ml) and brine $(10 \mathrm{ml})$, then dried $\left(\mathrm{MgSO}_{4}\right)$ and evaporated to give the benzoate (14)

(13 mg, 100\%), identical by NMR and TLC to the sample prepared from (13) (above). 

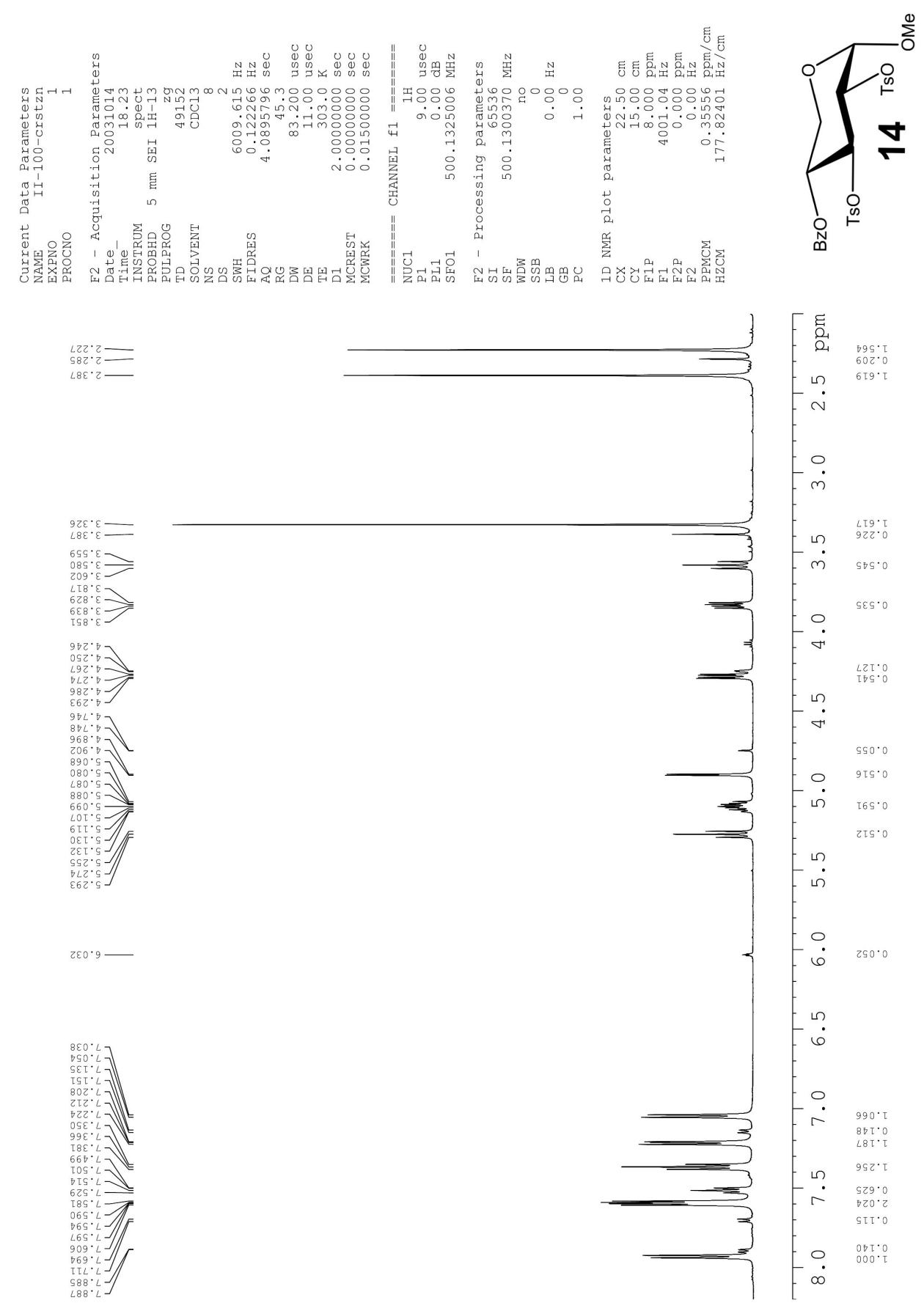


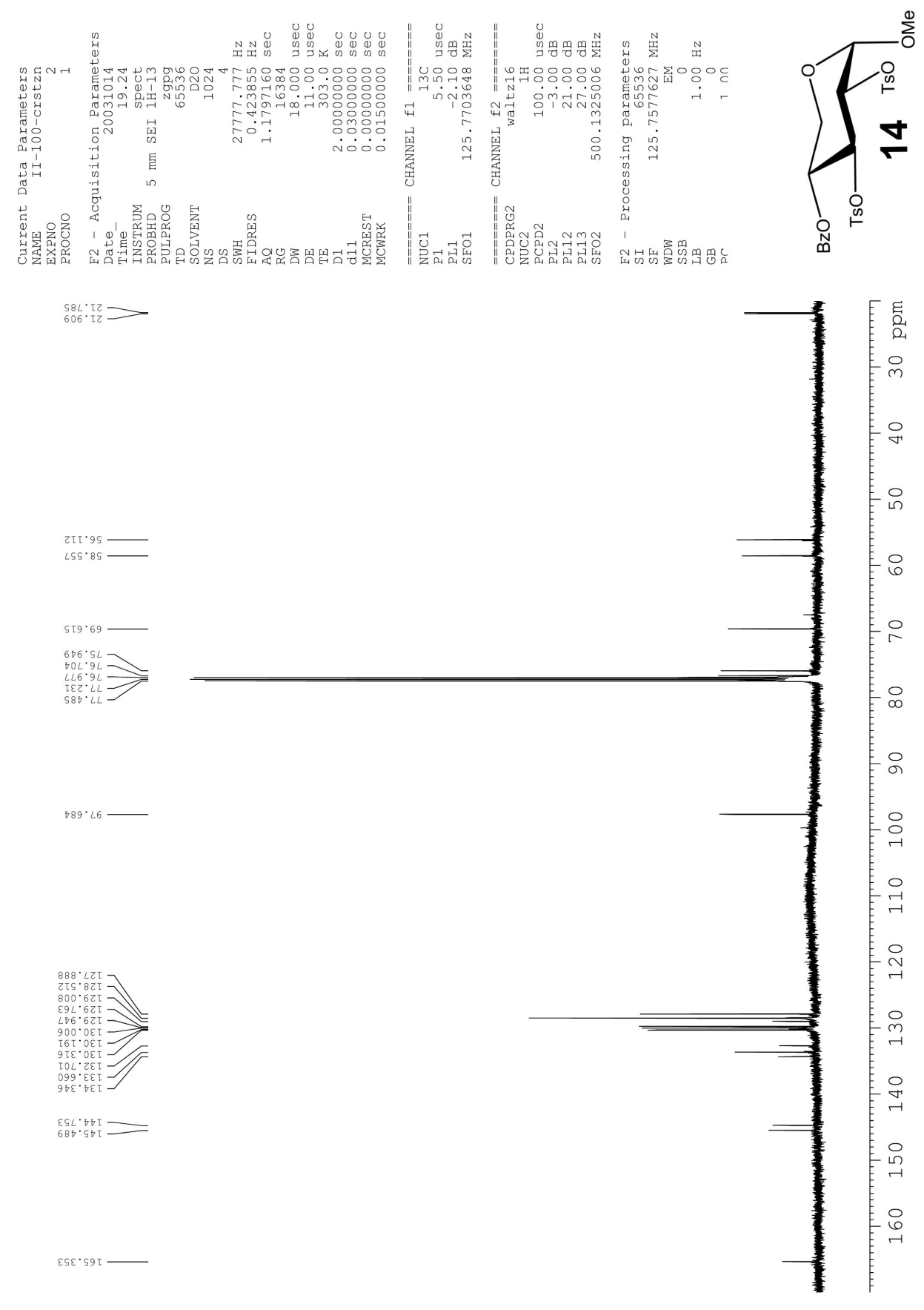




\section{Methyl 4-Azido-2,3-bis-(O-4-toluenesulfonyl)-4-deoxy- $\alpha$-D-xylopyranoside (15). A}

suspension of the tritosylate $(\mathbf{1 3})(200 \mathrm{mg}, 0.32 \mathrm{mmol})$ and sodium azide $(22 \mathrm{mg}, 3.06$ mmol) in dry DMF $(30 \mathrm{ml})$ was heated at $130{ }^{\circ} \mathrm{C}$ for 45 days. The cooled reaction mixture was evaporated and the residue was extracted with EtOAc $(45 \mathrm{ml})$. The organic extract was washed with $5 \% \mathrm{HCl}(2 \times 25 \mathrm{ml})$, saturated $\mathrm{NaHCO}_{3}(2 \times 25 \mathrm{ml})$ and brine $(10$ $\mathrm{ml})$, then dried $\left(\mathrm{MgSO}_{4}\right)$ and evaporated to a gum. This was purified by column chromatography (eluent: $\mathrm{EtOAc} / \mathrm{CHCl}_{3} /$ hexane 1:2:3) to give the title azido derivative (15) as a colorless gum (75 mg, 47\%). Recrystallization from EtOAc/hexane gave colorless crystals $(25 \mathrm{mg}, 13 \%)$, m.p. $55-57^{\circ} \mathrm{C} .[\alpha]_{\mathrm{D}} 81.0\left(c 0.5, \mathrm{CHCl}_{3}\right)$. ES-MS: 498 $(\mathrm{MH})^{+}, 515\left(\mathrm{M}+\mathrm{NH}_{4}\right)^{+}, 520(\mathrm{M}+\mathrm{Na})^{+} .{ }^{1} \mathrm{H} \mathrm{NMR}\left(500 \mathrm{MHz}, \mathrm{CDCl}_{3}\right): 2.41(\mathrm{~s}, 3 \mathrm{H}, \mathrm{Me})$; 2.42 (s, 3H, Me); 3.29 (s, 3H, OMe); 3.44-3.50 (m, 2H, H4,5e); 3.68 (dd, 1H, J=4.1, 9.7 Hz, H5a); 4.27 (dd, 1H, J=4.5, 9.7 Hz, H2); 4.83-4.88 (m, 2H, H1,3); 7.29 (br d, 2H, J= 8.4 Hz, Ar-H); 7.30 (br d, 2H, $J=8.6 \mathrm{~Hz}, \mathrm{Ar}-\mathrm{H}) ; 7.69$ (br d, 2H, $J=8.4 \mathrm{~Hz}, \mathrm{Ar}-\mathrm{H}) ; 7.79$

(br d, 2H, $J=8.3 \mathrm{~Hz}, \mathrm{Ar}-\mathrm{H}) .{ }^{13} \mathrm{C} \mathrm{NMR}\left(125 \mathrm{~Hz}, \mathrm{CDCl}_{3}\right): 22.1,22.2,56.3,59.8,63.3$, $61.2,76.0,98.0,128.6,128.7,130.2,130.3,133.2,134.4,145.4,145.8$. HRMS (TOF) calcd for $\mathrm{C}_{20} \mathrm{H}_{24} \mathrm{O}_{8} \mathrm{~S}_{2}(\mathrm{M}+\mathrm{H})^{+}$498.1005, found 498.0971. 

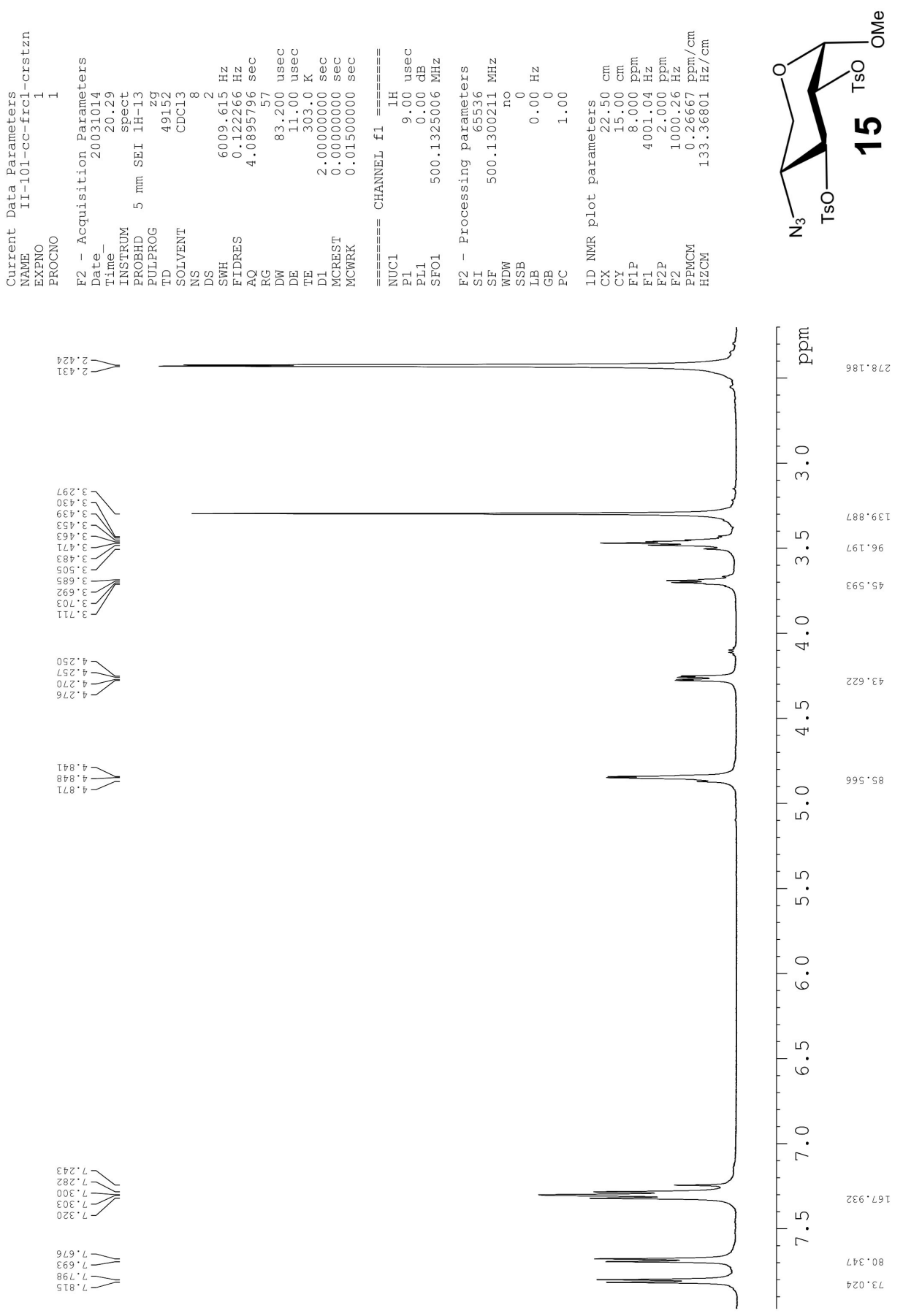

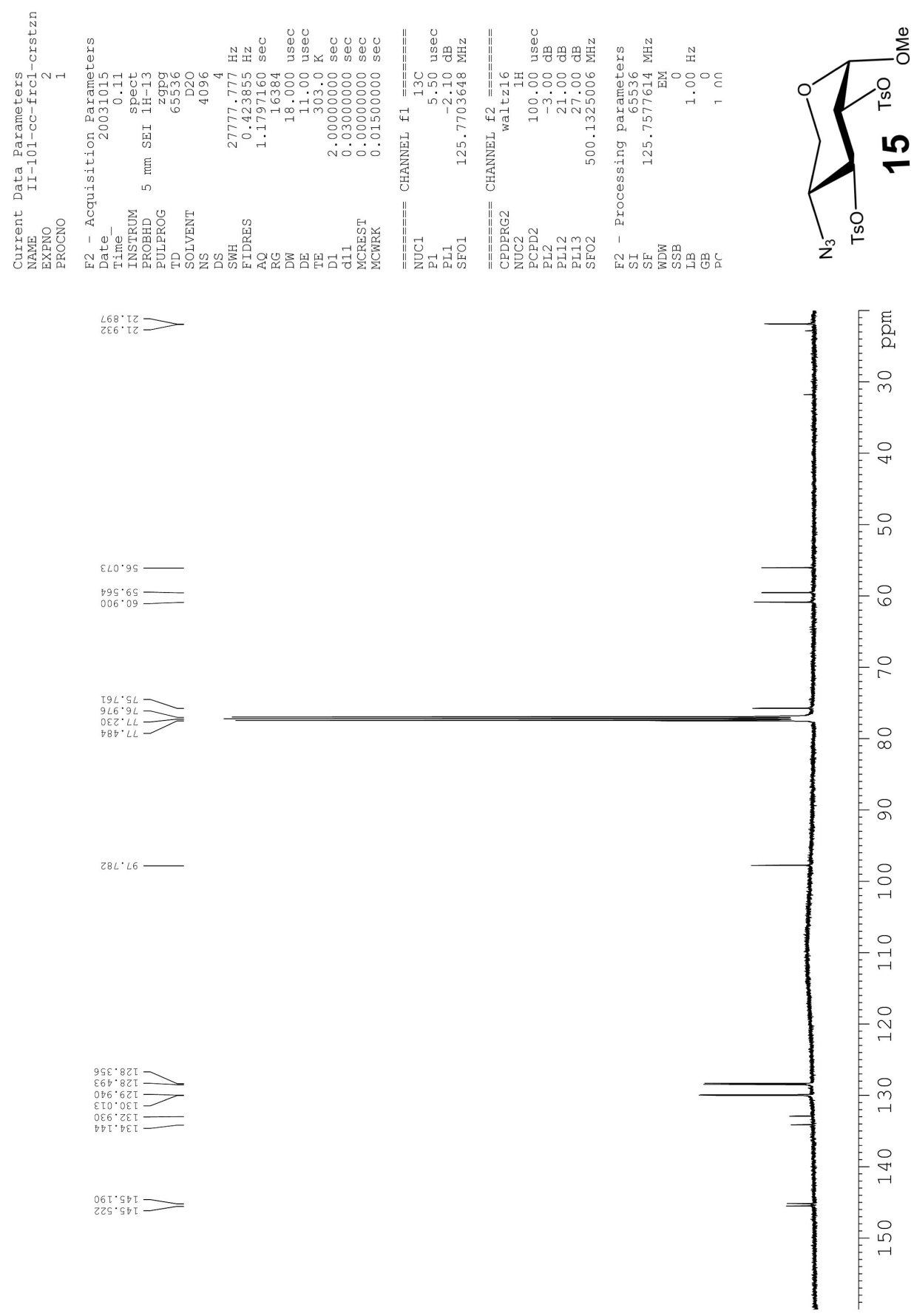
Methyl 2,3-Bis-(O-4-toluenesulfonyl)- $\alpha$-D-xylopyranoside (16). A suspension of the tritosylate (13) (200 mg, $319 \mathrm{mmol})$ and sodium nitrite $(220 \mathrm{mg}, 3.19 \mathrm{mmol})$ in dry DMF $(5 \mathrm{ml})$ was heated at $130{ }^{\circ} \mathrm{C}$ for 7 days. The cooled mixture was evaporated and the residue was taken up in EtOAc $(15 \mathrm{ml})$. The organic phase was washed with $5 \% \mathrm{HCl}$ (2x15 ml), saturated $\mathrm{NaHCO}_{3}(2 \times 15 \mathrm{ml})$ and brine $(10 \mathrm{ml})$, then dried $\left(\mathrm{MgSO}_{4}\right)$ and evaporated to give the ditosylate as colorless crystals (48 mg, 32\%), m.p. $152-154{ }^{\circ} \mathrm{C}$. Recrystallization from EtOAc/hexane raised the m.p. to $164-166{ }^{\circ} \mathrm{C}$ (lit. ${ }^{4}$ m.p. $168-169$ $\left.{ }^{\circ} \mathrm{C}\right) .[\alpha]_{\mathrm{D}} 59.5\left(c 0.1, \mathrm{CHCl}_{3}\right)\left(\right.$ lit. $^{4}[\alpha]_{\mathrm{D}} 60.3\left(c 1.7, \mathrm{CHCl}_{3}\right)$. ES-MS: $473(\mathrm{MH})^{+}, 490$ $\left(\mathrm{M}+\mathrm{NH}_{4}\right)^{+}, 495(\mathrm{M}+\mathrm{Na})^{+} .{ }^{1} \mathrm{H} \mathrm{NMR}\left(500 \mathrm{MHz}, \mathrm{CDCl}_{3}\right): 2.42(\mathrm{~s}, 3 \mathrm{H}, \mathrm{Me}) ; 2.44(\mathrm{~s}, 3 \mathrm{H}$, Me); 3.23 (s, 3H, OMe); 3.50 (br t, 1H, $J=11.0 \mathrm{~Hz}, \mathrm{H} 5 \mathrm{a}) ; 3.72(\mathrm{dd}, 1 \mathrm{H}, J=6.1,11.4 \mathrm{~Hz}$, H5b); 3.80-3.85 (m, 1H, H4); 4.28 (dd, 1H, J=3.6, 9.6 Hz, H2); 4.69 (d, 1H, J= 3.5 Hz, H1); $4.72(\mathrm{dd}, 1 \mathrm{H}, J=8.6,9.6 \mathrm{~Hz}, \mathrm{H} 3) ; 7.27$ (br d, 2H, $J=8.6 \mathrm{~Hz}, \mathrm{Ar}-\mathrm{H}) ; 7.31$ (br d, 2H, $J=8.6 \mathrm{~Hz}, \mathrm{Ar}-\mathrm{H}) ; 7.64$ (br d, $2 \mathrm{H}, J=8.3 \mathrm{~Hz}, \mathrm{Ar}-\mathrm{H}) ; 7.75$ (br d, 2H, $J=8.3 \mathrm{~Hz}, \mathrm{Ar}-\mathrm{H})$;

$\mathrm{OH}$ not observed. ${ }^{13} \mathrm{C}$ NMR $\left(125 \mathrm{~Hz}, \mathrm{CDC}_{3}\right): 21.9,22.0,55.7,60.9,69.3,75.8,80.9$, $97.3,128.2,128.6,130.0,130.1,132.5,133.3,145.4,145.7$. 


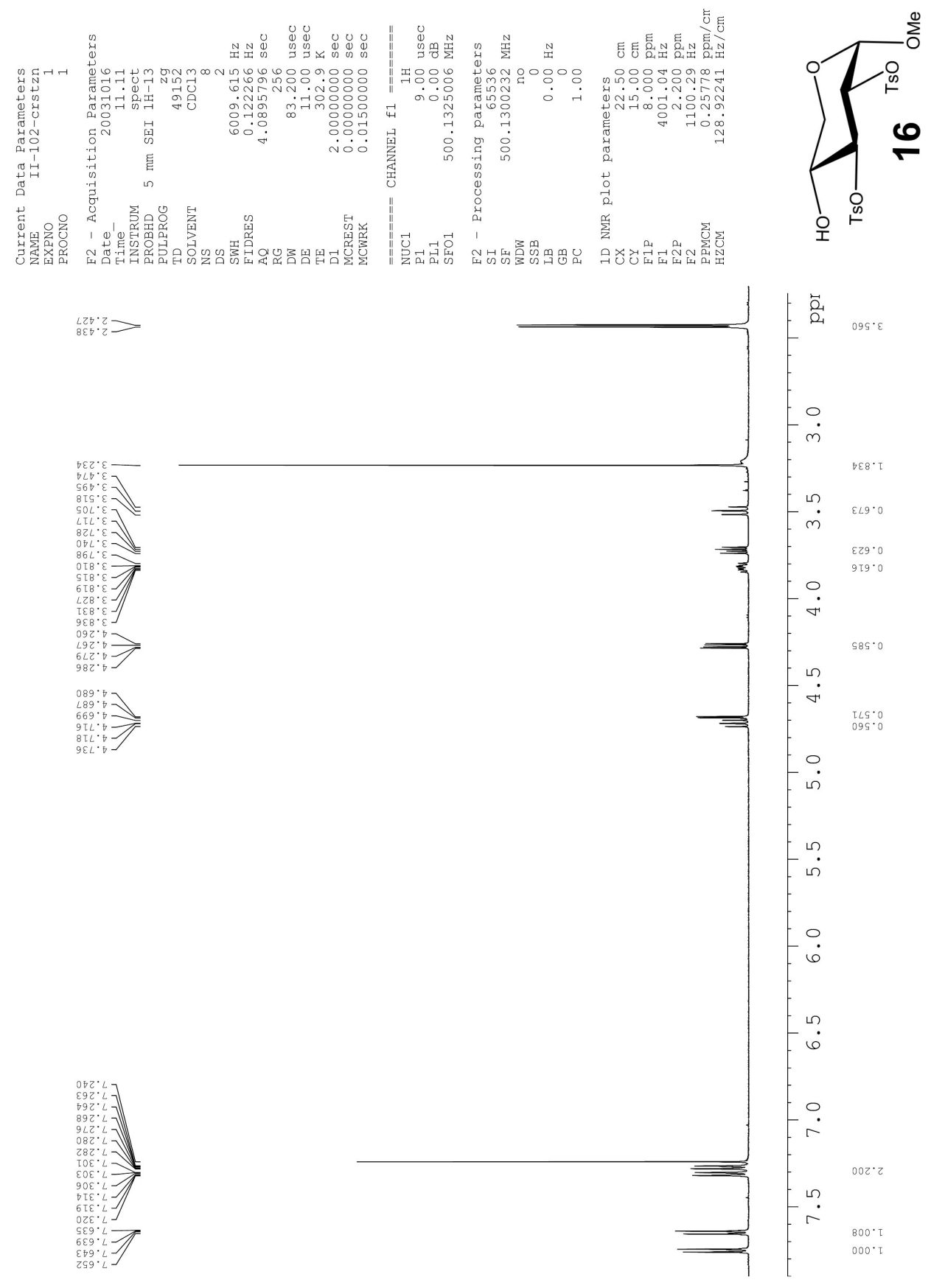




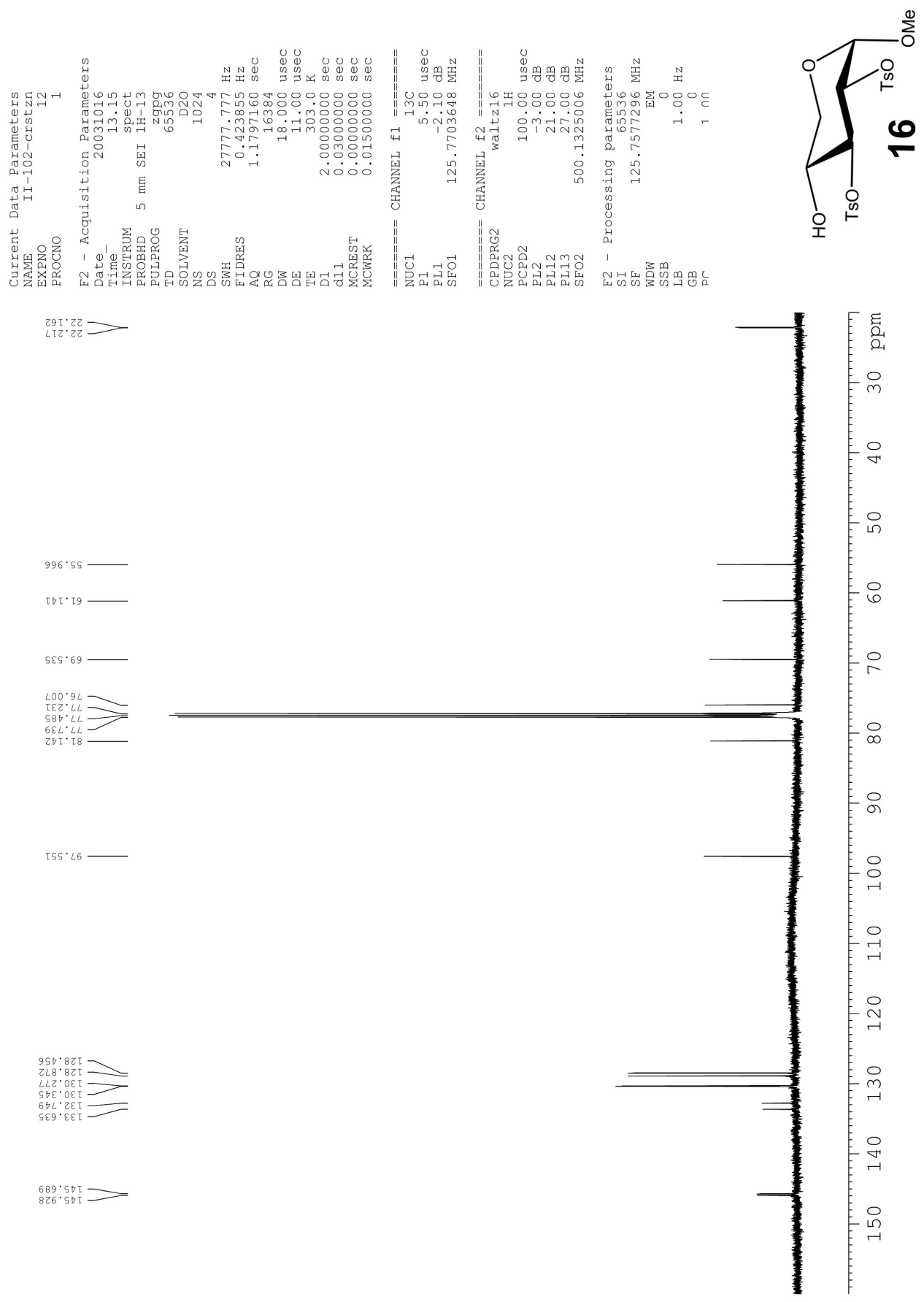


Methyl $\alpha$-D-Ribopyranoside (18). A solution of D-ribose (17) (2.00 g, $13.3 \mathrm{mmol})$ in $\mathrm{MeOH}(100 \mathrm{ml})$ was treated dropwise with acetyl chloride $(2.0 \mathrm{ml})$. The solution was then heated at $50{ }^{\circ} \mathrm{C}$ overnight. The cooled solution was neutralized by the addition of sodium methoxide solution, and then the mixture was filtered through Celite ${ }^{\circledR}$ and evaporated to a syrup (2.60 g) $R_{f}\left(3: 2: 1 \mathrm{CHCl}_{3} / \mathrm{EtOAc} / \mathrm{MeOH}\right): 0.23$.

A small sample of this was converted to its triacetate by treatment with acetic anhydride in pyridine. The ${ }^{1} \mathrm{H}$ NMR spectrum of the triacetate showed that mixture contained the $\alpha$ - and $\beta$-anomers in a ratio of 85:15. The crude mixture of anomers (18) was used directly for the next step.

Triacetate derivative ( $\alpha$-anomer): ${ }^{1} \mathrm{H}$ NMR (500 MHz, $\left.\mathrm{CDCl}_{3}\right): 2.00$ (s, 3H, OAc); 2.07 (s, 3H, OAc); 2.09 (s, 3H, OAc); 3.39 (s, 3H, OMe); 3.76 (dd, 1H, J=4.1, $12.6 \mathrm{~Hz}$, H5a); $3.96(\mathrm{dd}, 1 \mathrm{H}, J=2.7,12.6 \mathrm{~Hz}, \mathrm{H} 5 \mathrm{e}) ; 4.68(\mathrm{~d}, 1 \mathrm{H}, J=3.3 \mathrm{~Hz}, \mathrm{H} 1) ; 5.00(\mathrm{dt}, 1 \mathrm{H}, J=$ 0.9, 3.5 Hz, H4); 5.10-5.12 (m, 1H, H2); $5.34(\mathrm{t}, 1 \mathrm{H}, J=3.6 \mathrm{~Hz}, \mathrm{H} 3)$.

Methyl 2,3,4-Tris-(O-4-toluenesulfonyl)- $\alpha$-D-ribopyranoside (19). 4-Toluenesulfonyl chloride $(35.0 \mathrm{~g}, 184 \mathrm{mmol})$ was added portionwise to a stirred, chilled $\left(0{ }^{\circ} \mathrm{C}\right)$ solution of the crude mixture of anomers of (18) $(2.60 \mathrm{~g}, 13.3 \mathrm{mmol})$ in pyridine $(50 \mathrm{ml})$. After stirring for 5 days, the mixture was subjected to ultrasonication in a cleaning bath for $3 \mathrm{~h}$. The mixture was poured onto $100 \mathrm{~g}$ ice. After the ice had melted, the mixture was extracted with EtOAc $(2 \times 100 \mathrm{ml})$. The organic phase was washed with $5 \% \mathrm{HCl}(2 \times 100$ $\mathrm{ml})$, saturated $\mathrm{NaHCO}_{3}(2 \times 100 \mathrm{ml})$ and brine $(100 \mathrm{ml})$, then dried $\left(\mathrm{MgSO}_{4}\right)$ and evaporated to give a syrup. This was chromatographed on silica (eluent:

EtOAc/ $\mathrm{CHCl}_{3} /$ hexane $\left.1: 2: 3\right)$ to give the $\alpha$-tritosylate (19) as colorless syrup, 
contamininated with a little of the $\beta$-anomer $(2.04 \mathrm{~g}, 21 \%)$. A sample recrystallized twice from EtOAc/hexane gave the pure $\alpha$-isomer, m.p. $147-149{ }^{\circ} \mathrm{C} .[\alpha]_{\mathrm{D}} 31.0(c 0.5$, $\left.\mathrm{CHCl}_{3}\right)$. ES-MS: $627(\mathrm{MH})^{+}, 644\left(\mathrm{M}+\mathrm{NH}_{4}\right)^{+}, 649(\mathrm{M}+\mathrm{Na})^{+} .{ }^{1} \mathrm{H}$ NMR $(500 \mathrm{MHz}$, $\left.\mathrm{CDCl}_{3}\right): 2.41$ (s, 3H, Me); 2.43 (s, 6H, 2xMe); 3.18 (s, 3H, OMe); 3.80 (dd, $1 \mathrm{H}, J=4.5$, $11.0 \mathrm{~Hz}, \mathrm{H} 5 \mathrm{a}) ; 4.00(\mathrm{dd}, 1 \mathrm{H}, J=2.9,10.9 \mathrm{~Hz}, \mathrm{H} 5 \mathrm{~b}) ; 4.21(\mathrm{ddd}, 1 \mathrm{H}, J=2.9,4.4,7.4 \mathrm{~Hz}$, H4); $4.63(\mathrm{~d}, 1 \mathrm{H}, J=4.7 \mathrm{~Hz}, \mathrm{H} 2) ; 4.81$ (dd, 1H, $J=4.7,7.6 \mathrm{~Hz}, \mathrm{H} 3) ; 4.89$ (s, 1H, H1); 7.22-7.25 (m, 6H, Ar-H); 7.54 (br d, 2H, $J=8.3 \mathrm{~Hz}, \mathrm{Ar}-\mathrm{H}) ; 7.70($ br d, $2 \mathrm{H}, J=8.3 \mathrm{~Hz}$, Ar-H); 7.77 (br d, $2 \mathrm{H}, J=8.3 \mathrm{~Hz}, \mathrm{Ar}-\mathrm{H}) .{ }^{13} \mathrm{C}$ NMR $(125 \mathrm{~Hz}, \mathrm{CDCb}) 22.1,22.2,22.3$, 55.7, 68.0, 74.8, 77.3, 79.5, 105.6, 128.4, 128.5, 128.7, 130.4, 130.5, 130.7, 132.1, 132.9, 133.0, 145.6, 146.0, 146.5. HRMS (TOF) calcd for $\mathrm{C}_{27} \mathrm{H}_{30} \mathrm{O}_{11} \mathrm{~S}_{3} \mathrm{Na}(\mathrm{M}+\mathrm{Na})^{+}$649.0848, found 649.1091. HRMS (TOF) calcd for $\mathrm{C}_{26} \mathrm{H}_{27} \mathrm{O}_{10} \mathrm{~S}_{3}(\mathrm{M}+\mathrm{H}-\mathrm{MeOH})^{+}$595.0766, found 595.1001. 

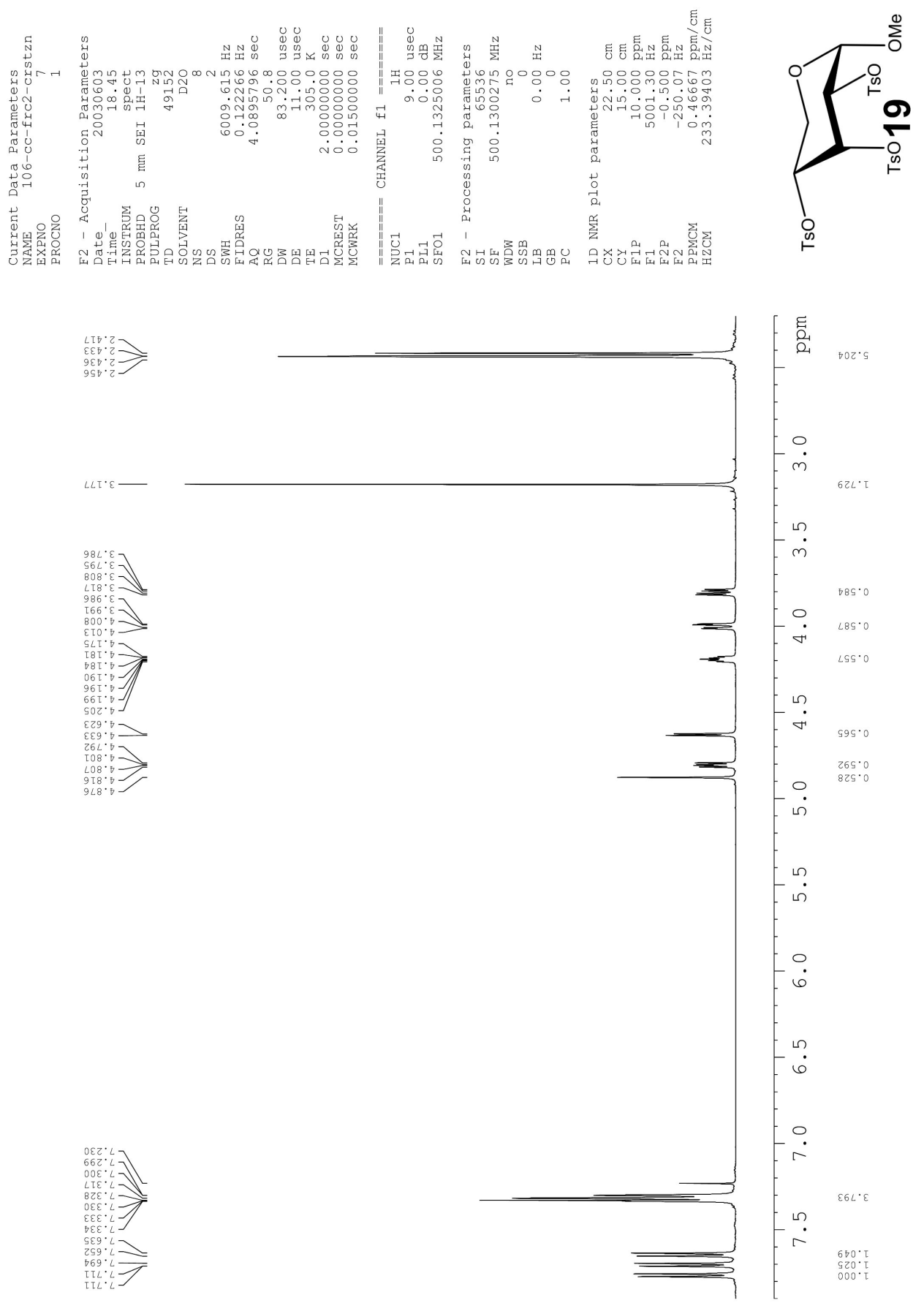


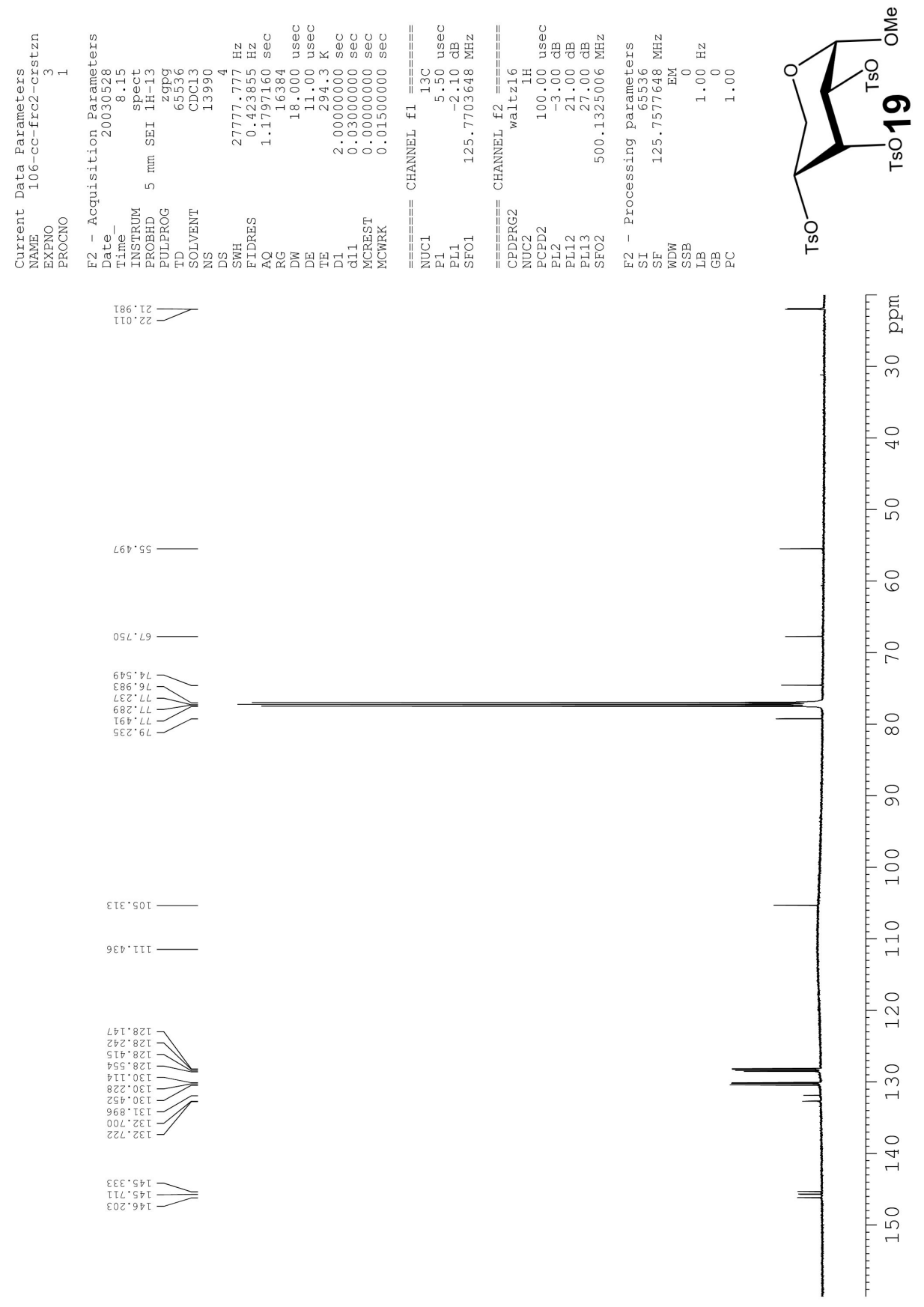




\section{Methyl 4-Benzoyl-2,3-bis-( $O$-4-toluenesulfonyl)- $\beta$-L-lyxopyranoside (20).}

(1) From (19): A suspension of the tritosylate (19) (30 mg, $0.048 \mathrm{mmol})$ and sodium benzoate (48 $\mathrm{mg}, 0.34 \mathrm{mmol}$ ) in $2.5 \mathrm{ml}$ of dry DMF was heated at $130{ }^{\circ} \mathrm{C}$ for $20 \mathrm{~h}$. The cooled reaction mixture was evaporated, and the residue was taken up into $50 \mathrm{ml}$ EtOAc and washed with $5 \% \mathrm{HCl}(2 \times 50 \mathrm{ml})$, saturated $\mathrm{NaHCO}_{3}(2 \times 50 \mathrm{ml})$ and brine $(50 \mathrm{ml})$, then dried $\left(\mathrm{MgSO}_{4}\right)$ and evaporated to give the benzoate (20) as a solid (21 $\left.\mathrm{mg}, 76 \%\right)$, m.p. $85-87{ }^{\circ} \mathrm{C}$. Recrystallization from EtOAc/hexane raised the m.p. to $90-92{ }^{\circ} \mathrm{C} .[\alpha]_{\mathrm{D}}$ $21.2\left(c\right.$ 0.2, $\left.\mathrm{CHCl}_{3}\right) . \mathrm{ES}-\mathrm{MS}: 577(\mathrm{MH})^{+}, 594\left(\mathrm{M}+\mathrm{NH}_{4}\right)^{+}, 599(\mathrm{M}+\mathrm{Na})^{+} .{ }^{1} \mathrm{H} \mathrm{NMR}(500$ $\left.\mathrm{MHz}, \mathrm{CDCl}_{3}\right): 2.27$ (s, 3H, Me); 2.47 (s, 3H, Me); 3.22 (s, 3H, OMe); 3.79 (dd, 1H, $J=$ 3.8, 13.1 Hz, H5a); 4.34-4.39 (m, 2H, H4,5b); 4.83 (d, 1H, J=4.4 Hz, H2); 5.00 (dd, 1H, $J=4.5,7.7 \mathrm{~Hz}, \mathrm{H} 3$ ); $5.02($ br s, 1H, H1 ); 7.09 (br d, 2H, $J=8.5 \mathrm{~Hz}, \mathrm{Ar}-\mathrm{H}) ; 7.41$ (br d, $2 \mathrm{H}, J=8.6 \mathrm{~Hz}, \mathrm{Ar}-\mathrm{H}) ; 7.45$ (br t, $2 \mathrm{H}, J=6.5 \mathrm{~Hz}, \mathrm{Ar}-\mathrm{H}) ; 7.60$ (br t, $1 \mathrm{H}, J=7.5 \mathrm{~Hz}, \mathrm{Ar}-$ H); 7.85 (br d, $2 \mathrm{H}, J=8.3 \mathrm{~Hz}, \mathrm{Ar}-\mathrm{H}) ; 7.92(\mathrm{brd}, 2 \mathrm{H}, J=8.3 \mathrm{~Hz}, \mathrm{Ar}-\mathrm{H}) .{ }^{13} \mathrm{C}$ NMR $(125$ $\mathrm{Hz}, \mathrm{CDCb}) 21.7,21.8,55.4,62.0,74.4,77.4,79.6,105.4,128.1,128.4,128.6,129.7$, 130.1, 131.9, 132.8, 133.3, 133.4, 145.5, 145.9, 165.6. HRMS (TOF) calcd for $\mathrm{C}_{27} \mathrm{H}_{29} \mathrm{O}_{10} \mathrm{~S}_{2}(\mathrm{M}+\mathrm{H})^{+}$577.1202, found 577.1201.

(2) From (22): A solution of the ditosylate (22) (13 $\mathrm{mg}, 0.028 \mathrm{mmol})$ in pyridine (3 $\mathrm{ml})$ was treated with benzoyl chloride $(0.15 \mathrm{ml}, 0.18 \mathrm{~g}, 1.3 \mathrm{mmol})$. After stirring overnight the solution was evaporated, then taken up in EtOAc $(10 \mathrm{ml})$. This was washed with $5 \% \mathrm{HCl}(3 \times 10 \mathrm{ml})$, saturated $\mathrm{NaHCO}_{3}(2 \times 10 \mathrm{ml})$ and brine $(10 \mathrm{ml})$, then dried $\left(\mathrm{MgSO}_{4}\right)$ and evaporated to give the benzoate (20) (10 mg, 63\%), identical by NMR and TLC to that prepared from (19) (above). 


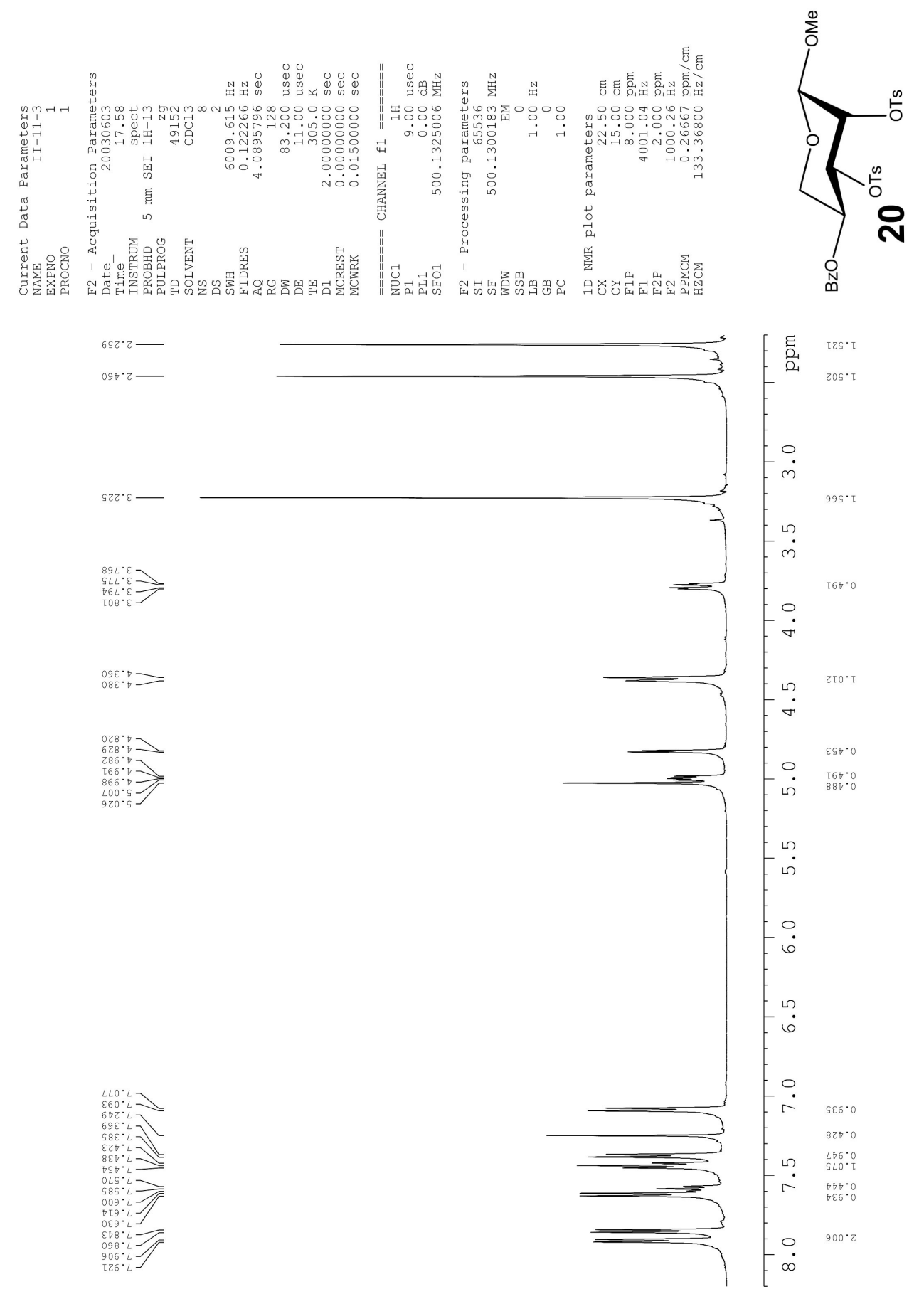




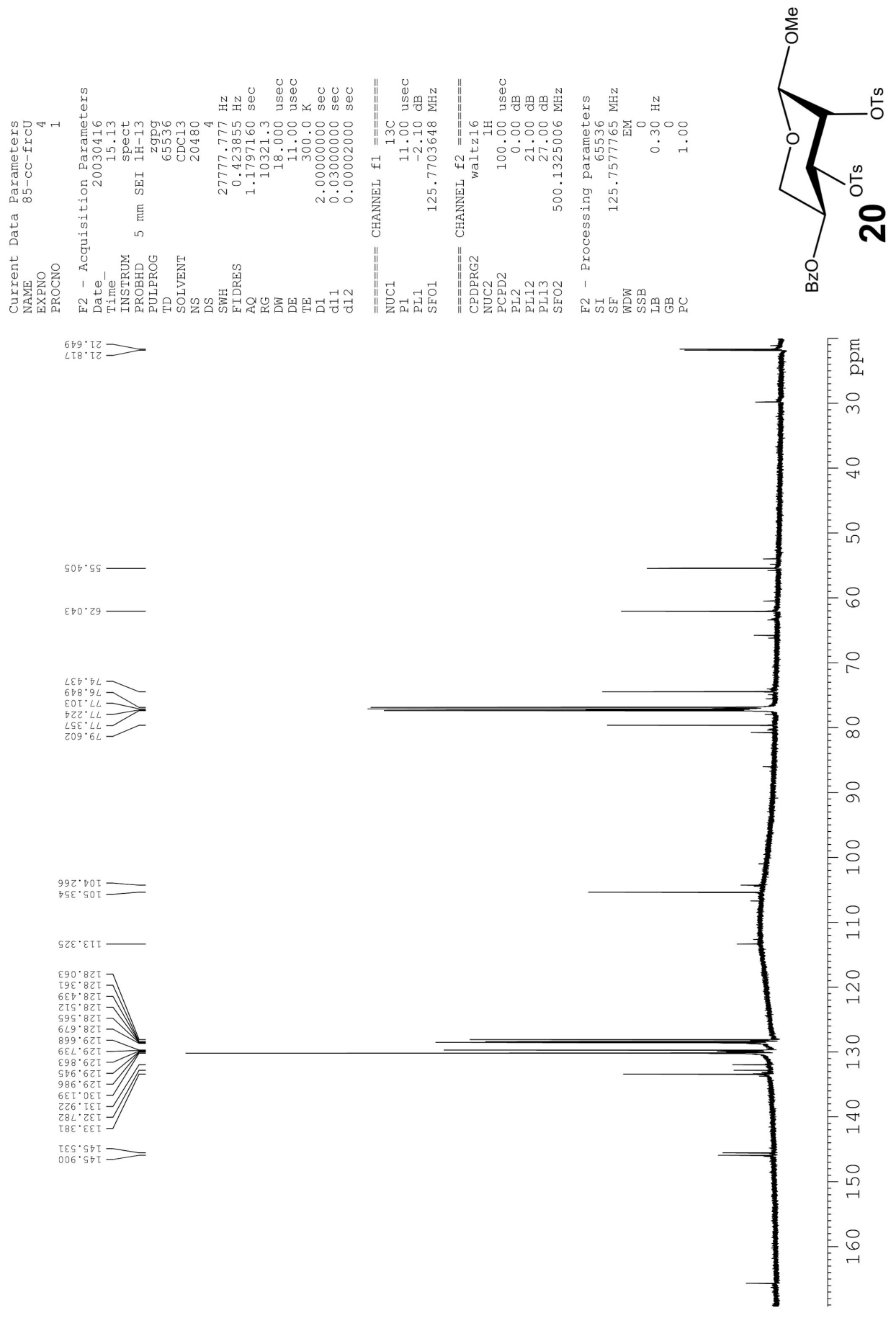




\section{Methyl 4-Azido-2,3-bis-(O-4-toluenesulfonyl)-4-deoxy- $\beta$-L-lyxopyranoside (21). A}

suspension of the tritosylate (19) $(30 \mathrm{mg}, 0.048 \mathrm{mmol})$ and sodium azide (28 $\mathrm{mg}, 0.43$ mmol) in $2.5 \mathrm{ml}$ of dry DMF was heated at $130{ }^{\circ} \mathrm{C}$ for $40 \mathrm{~h}$. The cooled reaction mixture was evaporated, and the residue was taken up into $50 \mathrm{ml}$ EtOAc and washed with 5\% $\mathrm{HCl}(2 \times 50 \mathrm{ml})$, saturated $\mathrm{NaHCO}_{3}(2 \times 50 \mathrm{ml})$ and brine $(50 \mathrm{ml})$, then dried $\left(\mathrm{MgSO}_{4}\right)$ and evaporated to give the azide (21) as a solid (16 mg, 67\%), m.p. $110-115^{\circ} \mathrm{C} .[\alpha]_{\mathrm{D}} 70.8(c$ 0.6, $\left.\mathrm{CHCl}_{3}\right) . \mathrm{ES}-\mathrm{MS}: 498(\mathrm{MH})^{+}, 515\left(\mathrm{M}+\mathrm{NH}_{4}\right)^{+}, 520(\mathrm{M}+\mathrm{Na})^{+} .{ }^{1} \mathrm{H}$ NMR $(500 \mathrm{MHz}$, $\left.\mathrm{CDCl}_{3}\right): 2.44$ (s, 3H, Me); 2.45 (s, 3H, Me); 3.04 (dd, 1H, J=4.7, $\left.13.4 \mathrm{~Hz}, \mathrm{H} 5 \mathrm{a}\right) ; 3.33$ (s, 3H, OMe); 3.39 (dd, 1H, J = 3.0, $13.4 \mathrm{~Hz}, \mathrm{H} 5 \mathrm{~b}) ; 4.20$ (ddd, 1H, J=3.1, 4.7, 7.8 Hz, H4); $4.67(\mathrm{~d}, 1 \mathrm{H}, J=4.7, \mathrm{H} 2) ; 4.82(\mathrm{dd}, 1 \mathrm{H}, J=4.7,7.8 \mathrm{~Hz}, \mathrm{H} 3) ; 4.95$ (br s, 1H, H1); 7.32 (br d, $2 \mathrm{H}, J=8.0 \mathrm{~Hz}, \mathrm{Ar}-\mathrm{H}) ; 7.34$ (br d, $2 \mathrm{H}, J=8.0 \mathrm{~Hz}, \mathrm{Ar}-\mathrm{H}) ; 7.69$ (br d, $2 \mathrm{H}, J=8.3 \mathrm{~Hz}$, $\mathrm{Ar}-\mathrm{H}) ; 7.79$ (br d, $2 \mathrm{H}, J=8.3 \mathrm{~Hz}, \mathrm{Ar}-\mathrm{H}) .{ }^{13} \mathrm{C} \mathrm{NMR}\left(125 \mathrm{~Hz}, \mathrm{CDCl}_{3}\right)$ 21.9, 22.0, 51.6, $56.2,75.1,78.5,79.6,106.3,128.3,128.4,130.2,130.3,132.4,133.0,145.7,146.0$. HRMS (TOF) calcd for $\mathrm{C}_{20} \mathrm{H}_{24} \mathrm{~N}_{3} \mathrm{O}_{8} \mathrm{~S}_{2}(\mathrm{M}+\mathrm{H})^{+}$498.1005, found 498.1182. 

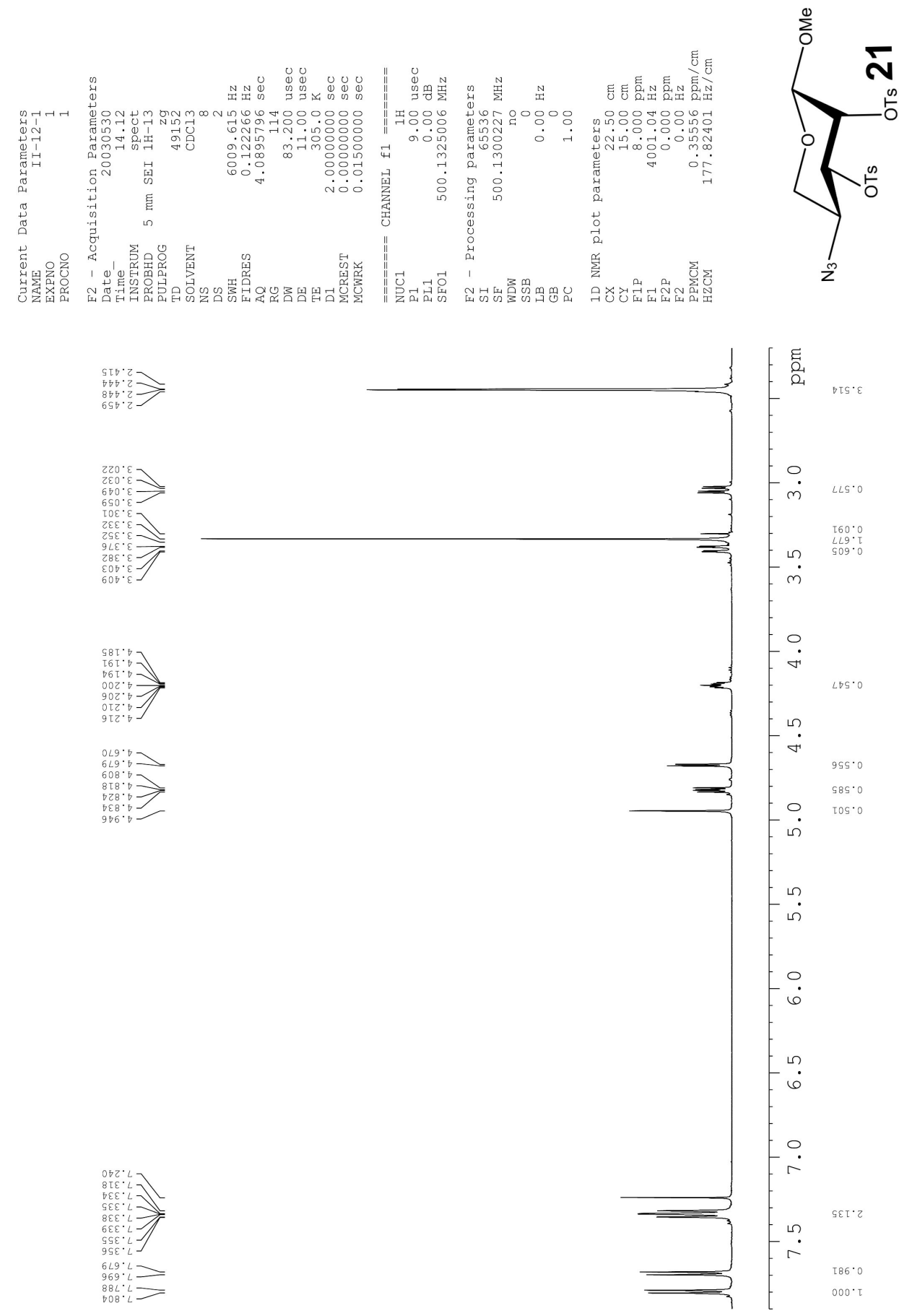


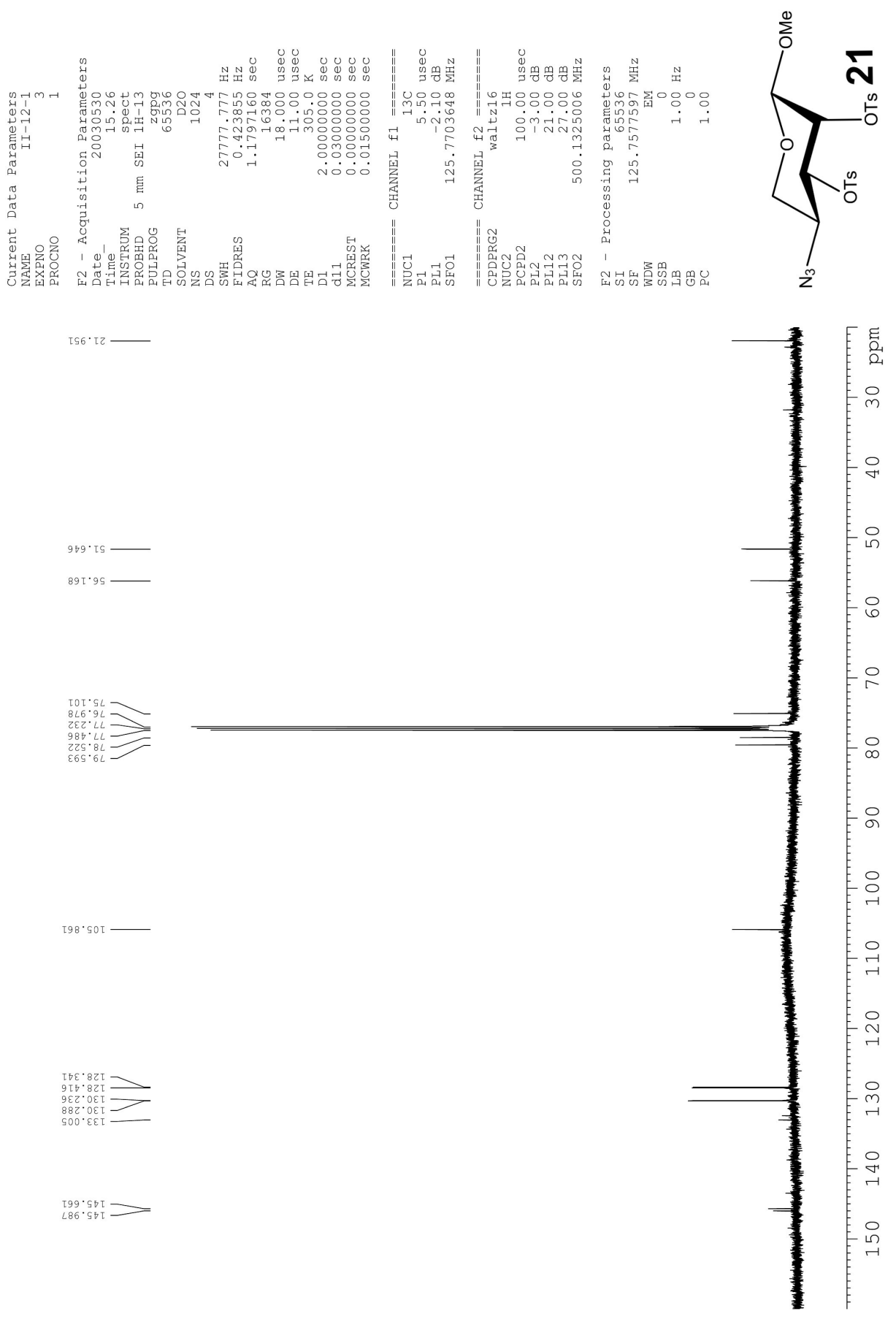


Methyl 2,3-Bis-(O-4-toluenesulfonyl)- $\beta$-L-lyxopyranoside (22). A suspension of the tritosylate (21) (50 mg, $0.080 \mathrm{mmol})$ and sodium nitrite $(55 \mathrm{mg}, 0.80 \mathrm{mmol})$ in dry DMF (5 ml) was heated at $130{ }^{\circ} \mathrm{C}$ for $48 \mathrm{~h}$. The cooled mixture was evaporated and the residue was extracted with EtOAc $(25 \mathrm{ml})$. The organic extract was washed with $5 \% \mathrm{HCl}(2 \times 10$ $\mathrm{ml})$, saturated $\mathrm{NaHCO}_{3}(2 \times 10 \mathrm{ml})$ and brine $(10 \mathrm{ml})$, then dried $\left(\mathrm{MgSO}_{4}\right)$ and evaporated to a gum. This was triturated with hexane to give the ditosylate (22) as a colorless oil (30 mg, 81\%). $[\alpha]_{\mathrm{D}} 15.3\left(c 0.2, \mathrm{CHCl}_{3}\right) . R_{f}\left(1: 2: 3 \mathrm{EtOAc} / \mathrm{CHCl}_{3} /\right.$ hexane): 0.15. ES-MS: $473(\mathrm{MH})^{+}, 490\left(\mathrm{M}+\mathrm{NH}_{4}\right)^{+}, 495(\mathrm{M}+\mathrm{Na})^{+} .{ }^{1} \mathrm{H} \mathrm{NMR}\left(500 \mathrm{MHz}, \mathrm{CDCl}_{3}\right): 2.43(\mathrm{~s}, 3 \mathrm{H}$, Me); 2.45 (s, 3H, Me); 3.28 (br d, 1H, J=12.2 Hz, H5a); 3.32 (s, 3H, OMe); 3.64 (dd, $1 \mathrm{H}, J=2.3,12.4 \mathrm{~Hz}, \mathrm{H} 5 \mathrm{~b}) ; 4.19-4.21$ (m, 1H, H4); 4.69 (d, 1H, $J=4.7 \mathrm{~Hz}, \mathrm{H} 1) ; 4.93-$ $4.96(\mathrm{~m}, 2 \mathrm{H}, \mathrm{H} 2,3) ; 7.31$ (br d, 2H, $J=8.1 \mathrm{~Hz}, \mathrm{Ar}-\mathrm{H}) ; 7.35$ (br d, 2H, $J=8.2 \mathrm{~Hz}, \mathrm{Ar}-\mathrm{H})$; 7.70 (br d, 2H, $J=8.1 \mathrm{~Hz}$, Ar-H); 7.80 (br d, 2H, $J=8.1 \mathrm{~Hz}$, Ar-H); OH not observed. ${ }^{13} \mathrm{C}$ NMR $\left(125 \mathrm{~Hz}, \mathrm{CDCl}_{3}\right)$ 21.8, 21.8, 56.1, 61.1, 74.5, 79.8, 81.0, 106.0, 128.2, 128.2, 130.1, 130.2, 132.5, 132.8, 145.5, 145.7. HRMS (TOF) calcd for $\mathrm{C}_{20} \mathrm{H}_{25} \mathrm{O}_{9} \mathrm{~S}_{2}(\mathrm{M}+\mathrm{H})^{+}$ 473.0940, found 473.0900. 


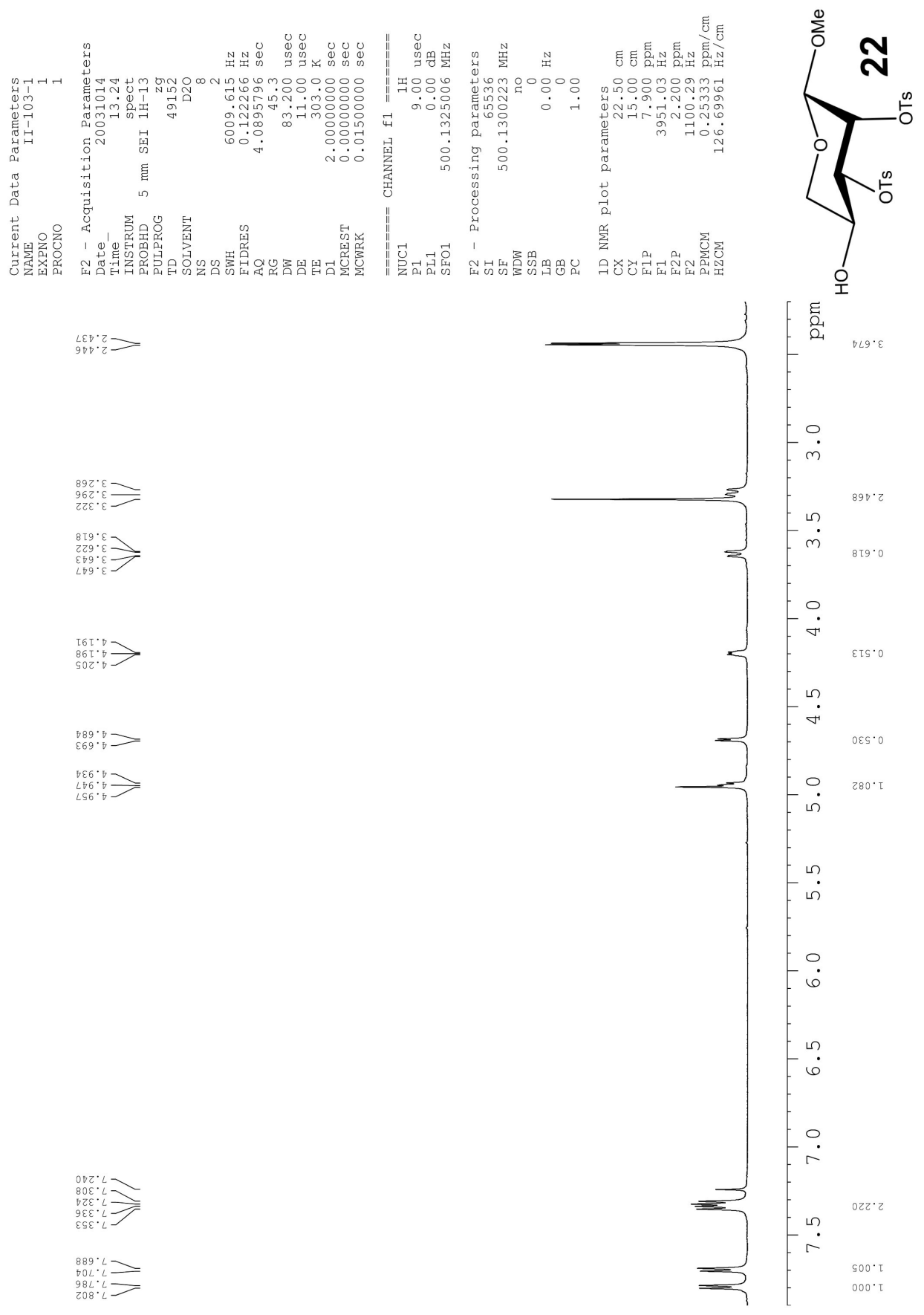




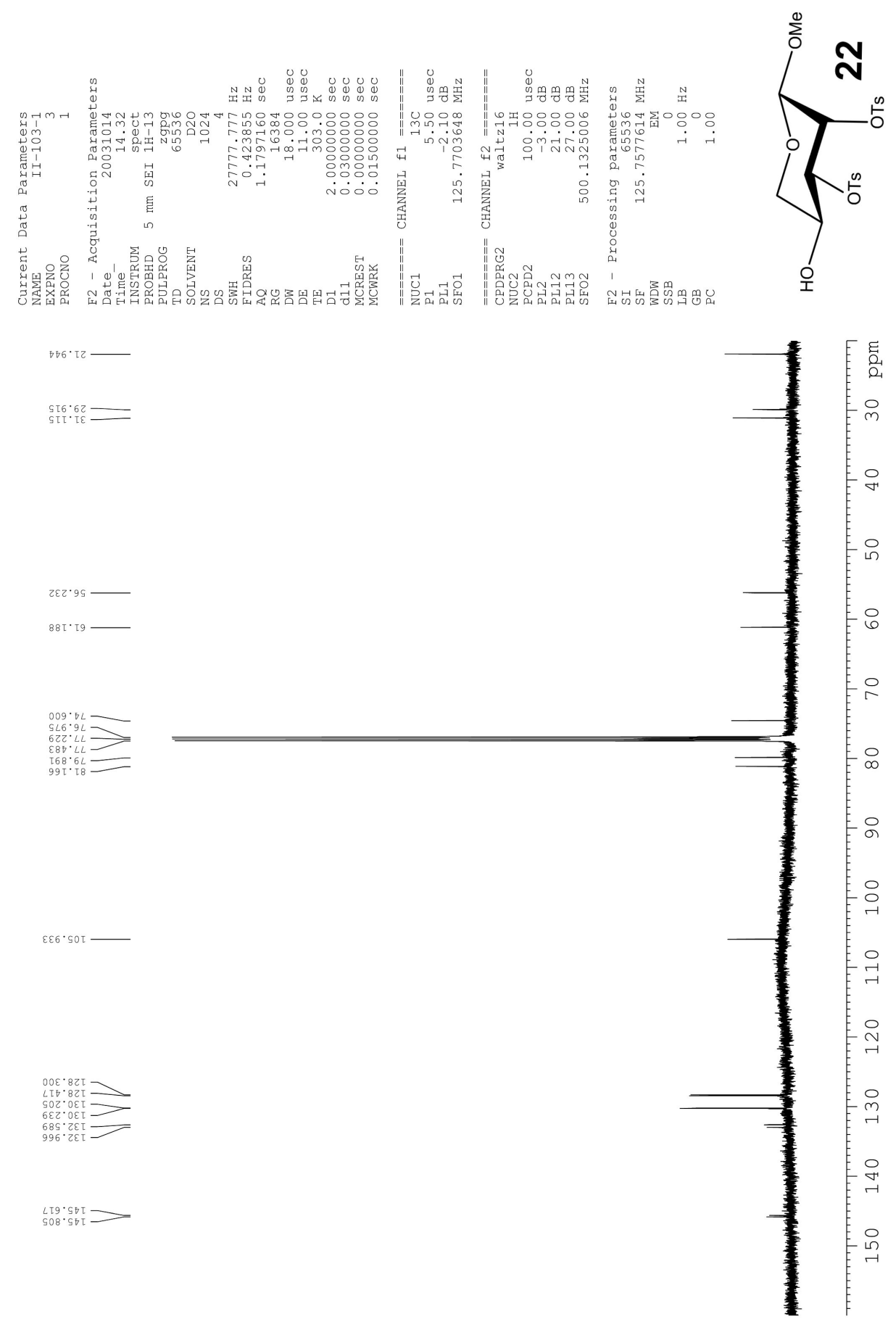


Methyl $\alpha$-D-Lyxopyranoside (24). Acetyl chloride (1.0 ml, $14 \mathrm{mmol}$ ) was added dropwise to a stirred solution of D-lyxose $(\mathbf{2 3})(1.00 \mathrm{~g}, 6.6 \mathrm{mmol})$ in dry $\mathrm{MeOH}(50 \mathrm{ml})$. The solution was heated overnight at $50^{\circ} \mathrm{C}$, and then cooled in ice. The solution was then neutralized by the addition of solid $\mathrm{Ag}_{2} \mathrm{CO}_{3}$. Stirring was continued for $2 \mathrm{~h}$ at this temperature, then the mixture was filtered through Celite ${ }^{\circledR}$ and the filtrate evaporated to give the methyl pyranoside (24) as a syrup, in quantitative yield. The crude product was used directly for subsequent steps. Crystals slowly formed on standing, m.p. $91-93{ }^{\circ} \mathrm{C}$ (lit. m.p. ${ }^{6} 102-103{ }^{\circ} \mathrm{C}$; lit. m.p. $\left.{ }^{9} 108-109^{\circ} \mathrm{C}\right) . \quad R_{f}(3: 2: 1 \mathrm{CHCl} / \mathrm{EtOAc} / \mathrm{MeOH}): 0.23$.

A small sample of the crude syrup was acetylated with $\mathrm{Ac}_{2} \mathrm{O} /$ pyridine gave the triacetate derivative, which was shown by ${ }^{1} \mathrm{H}$ NMR spectroscopy to be an $87: 13$ mixture of the $\alpha / \beta$ anomers. ${ }^{1} \mathrm{H}$ NMR $\left(500 \mathrm{MHz}, \mathrm{CDCl}_{3}\right)$ : ( $\alpha$-anomer) 1.98 (s, 3H, OAc); 2.01 (s, 3H, OAc); 2.09 (s, 3H, OAc); 3.37 (s 3H, OMe); 3.58 (dd, 1H, $J=9.3,11.0 \mathrm{~Hz}, \mathrm{H} 5$ ); 3.85 (dd, $1 \mathrm{H}, J=5.4,11.0 \mathrm{~Hz}, \mathrm{H} 5) ; 4.61(\mathrm{~d}, 1 \mathrm{H}, J=2.4 \mathrm{~Hz}, \mathrm{H1}) ; 5.17(\mathrm{dd}, 1 \mathrm{H}, J=5.4,9.7$ $\mathrm{Hz}, \mathrm{H} 4) ; 5.19(\mathrm{dd}, 1 \mathrm{H}, J=2.5,3.4 \mathrm{~Hz}, \mathrm{H} 2) ; 5.31(\mathrm{dd}, 1 \mathrm{H}, J=3.5,10.3 \mathrm{~Hz}, \mathrm{H} 3)$.

Methyl 2,3,4-Tris-(O-4-toluenesulfonyl)- $\alpha$-D-lyxopyranoside (25). A stirred, chilled $\left(0{ }^{\circ} \mathrm{C}\right)$ solution of the crude methyl $\alpha$-D-lyxopyranoside (24) $(5.00 \mathrm{~g}, 30.5 \mathrm{mmol})$ in pyridine $(50 \mathrm{ml})$ was treated portionwise during $30 \mathrm{~min}$ with 4-toluenesulfonyl chloride (40.0 g, $210 \mathrm{mmol}$ ). After stirring for 5 days at room temperature, the mixture was sonicated in a cleaning bath for $3 \mathrm{~h}$, and then poured onto $100 \mathrm{~g}$ ice. After the ice had melted, the mixture was extracted into EtOAc $(200 \mathrm{ml})$ and washed with $5 \% \mathrm{HCl}(2 \times 100$ $\mathrm{ml})$, saturated $\mathrm{NaHCO}_{3}(2 \times 100 \mathrm{ml})$ and brine $(100 \mathrm{ml})$, then dried $\left(\mathrm{MgSO}_{4}\right)$ and 
evaporated to a syrup. This was triturated with hexane, and then the residue was purified by column chromatography (eluent: $\mathrm{EtOAc} / \mathrm{CHCl}_{3} /$ hexane $1: 2: 3$ ) to give the tritosylated lyxose (25) (10.9 g, 57\%), which was shown by ${ }^{1} \mathrm{H}$ NMR spectroscopy to be contaminated with $7 \%$ of the $\beta$-anomer. Two recrystallizations from EtOAc/hexane gave the pure tritosylate $(\mathbf{2 5})$ as colorless crystals, m.p. $66-68{ }^{\circ} \mathrm{C} .[\alpha]_{\mathrm{D}} 7.2\left(c 1.0, \mathrm{CHC}_{3}\right)$. ES-MS: $627(\mathrm{MH})^{+}, 644\left(\mathrm{M}+\mathrm{NH}_{4}\right)^{+}, 649(\mathrm{M}+\mathrm{Na})^{+} .{ }^{1} \mathrm{H}$ NMR $\left(500 \mathrm{MHz}, \mathrm{CDCl}_{3}\right): 2.41$ (s, 3H, Me); 2.42 (s, 3H, Me); 2.44 (s, 3H, Me); 3.28 (s, 3H, OMe); 3.45-3.54 (m, 1H, H5a); $3.74(\mathrm{dd}, 1 \mathrm{H}, J=4.6,11.3 \mathrm{~Hz}, \mathrm{H} 5 \mathrm{~b}) ; 4.62(\mathrm{dd}, 1 \mathrm{H}, J=3.1,9.0 \mathrm{~Hz}, \mathrm{H} 3)$; 4.63-4.74 (m, 3H, H1,2,4); 7.26 (br d, 2H, $J=8.1 \mathrm{~Hz}$, Ar-H); 7.31 (br d, 2H, J=8.0 Hz, Ar-H); 7.34 (br d, $2 \mathrm{H}, J=8.2 \mathrm{~Hz}, \mathrm{Ar}-\mathrm{H}) ; 7.58$ (br d, $2 \mathrm{H}, J=8.2 \mathrm{~Hz}, \mathrm{Ar}-\mathrm{H}) ; 7.68$ (br d, $2 \mathrm{H}, J=$ 8.0 Hz, Ar-H); 7.75 (br d, $2 \mathrm{H}, J=8.0 \mathrm{~Hz}, \mathrm{Ar}-\mathrm{H}) .{ }^{13} \mathrm{C} \mathrm{NMR}\left(125 \mathrm{~Hz}, \mathrm{CDCl}_{3}\right)$ 22.4, 56.4, $61.1,72.9,74.2,76.9,99.0,128.8,128.9,129.0,130.5,130.6,130.7,132.8,133.3,133.6$, 146.0, 146.5. HRMS (TOF) calcd for $\mathrm{C}_{27} \mathrm{H}_{31} \mathrm{O}_{11} \mathrm{~S}_{3}(\mathrm{M}+\mathrm{H})^{+}$627.1029, found 627.1073. 

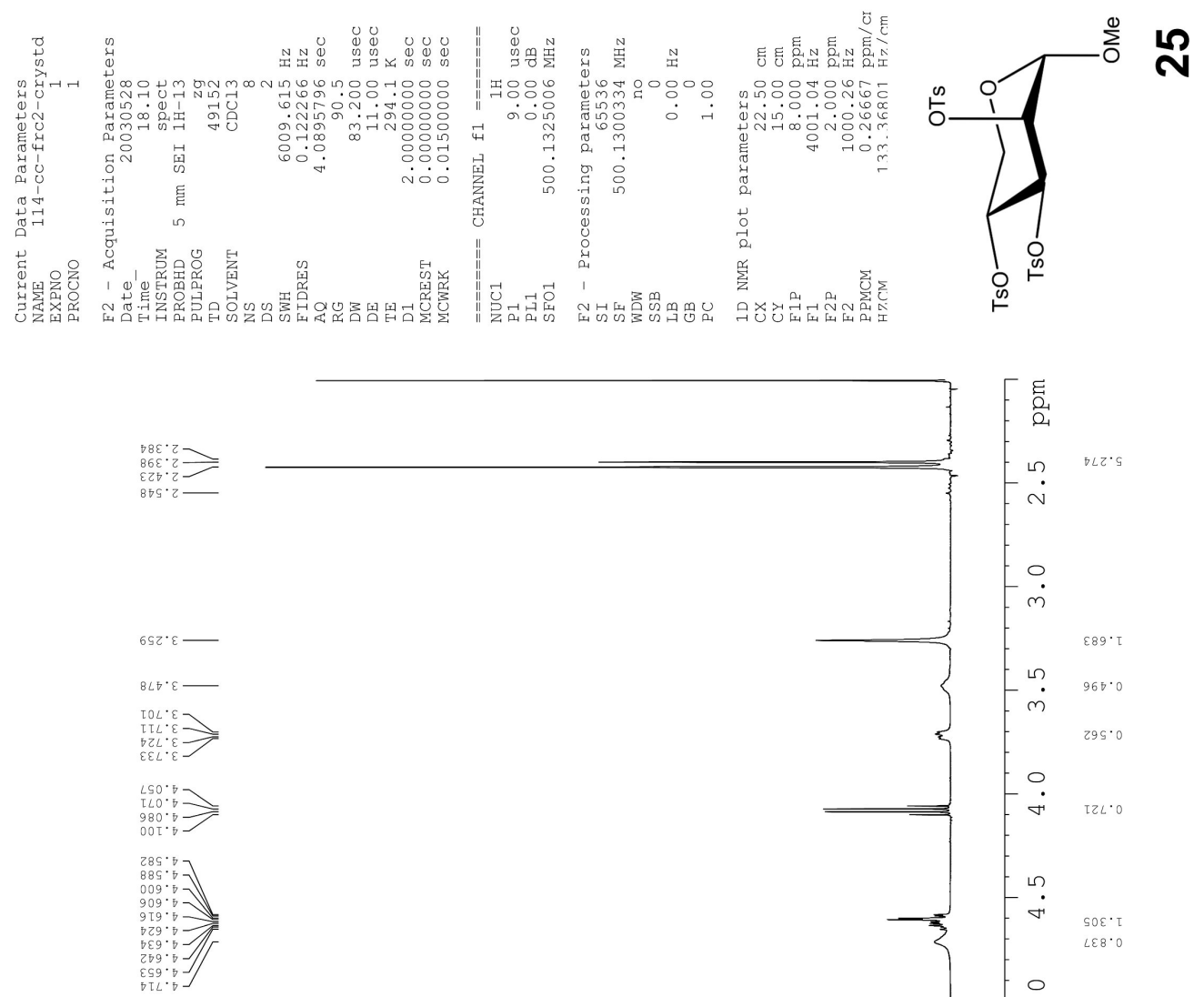

탱

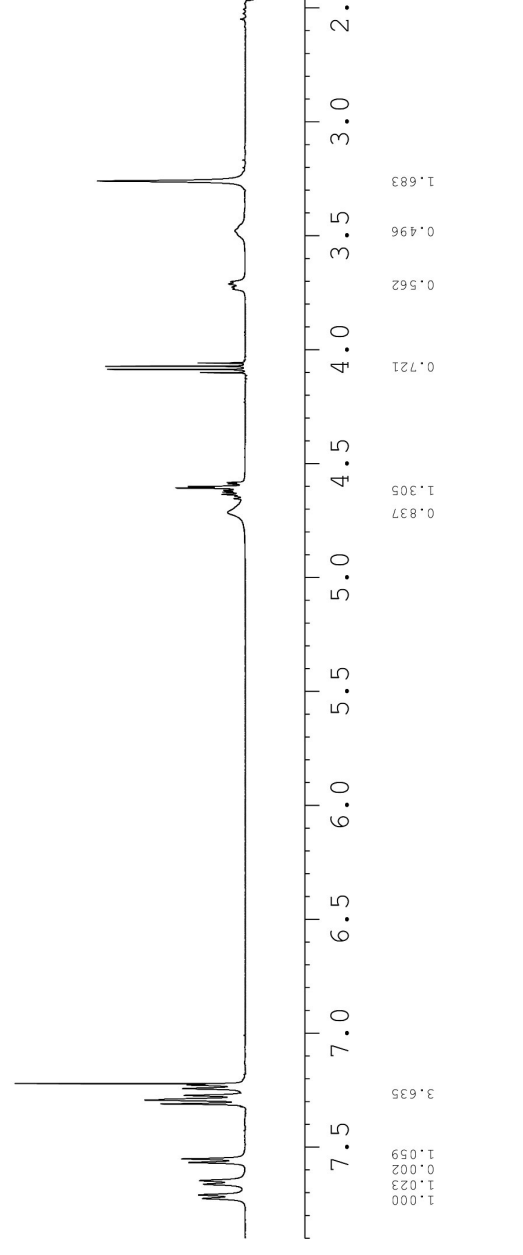




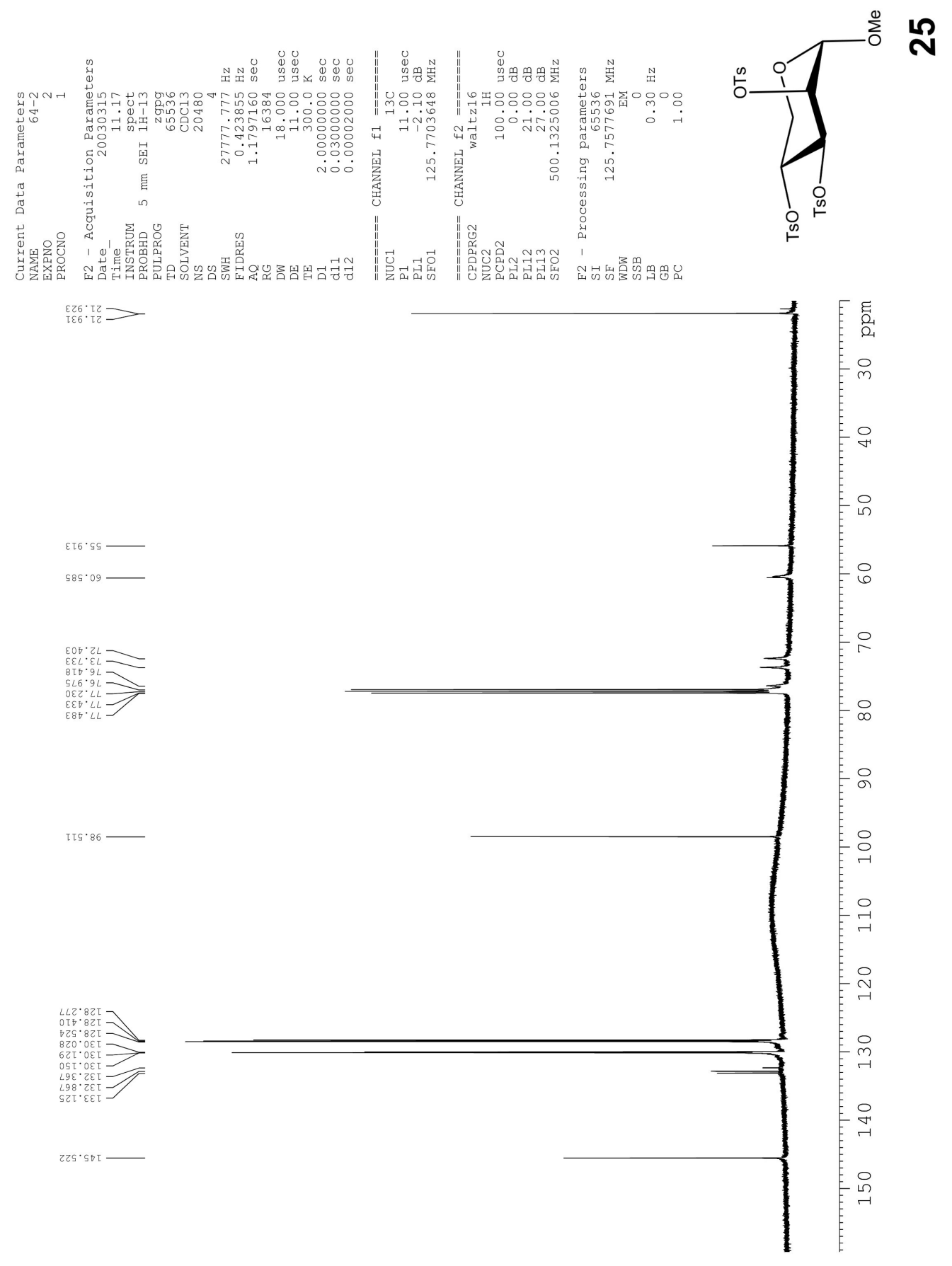


3R,6S-3-Benzoyloxy-6-methoxy-4-(4-toluenesulfonyloxy)-3,6-dihydro-2H-pyran (26). A mixture of the pure tritosylated lyxose $(\mathbf{2 5})(80 \mathrm{mg}, 0.13 \mathrm{mmol})$ and sodium benzoate (250 mg, $1.74 \mathrm{mmol})$ in DMF (20 ml) was heated at $130{ }^{\circ} \mathrm{C}$ for 5 days. The cooled mixture was evaporated then the residue was extracted into EtOAc $(50 \mathrm{ml})$ and filtered. The filtrate was washed with $5 \% \mathrm{HCl}(2 \times 50 \mathrm{ml})$, saturated $\mathrm{NaHCO}_{3}(2 \times 50 \mathrm{ml})$ and brine (50 ml), then dried $\left(\mathrm{MgSO}_{4}\right)$ and evaporated. The residue was purified by flash chromatography to give the dihydropyran (26) as a colorless oil (15 mg, 29\%). $[\alpha]_{\mathrm{D}} 10.3$ $\left(c\right.$ 1.2, $\left.\mathrm{CHCl}_{3}\right)$. ES-MS: $405(\mathrm{MH})^{+}, 422\left(\mathrm{M}+\mathrm{NH}_{4}\right)^{+}, 427(\mathrm{M}+\mathrm{Na})^{+} .{ }^{1} \mathrm{H} \mathrm{NMR}(500 \mathrm{MHz}$, $\mathrm{CDCl}_{3}$ ): (carbohydrate numbering) 2.32 (s, 3H, Me); 3.40 (s, 3H, OMe); 3;87 (dd, 1H, J $=1.1,13.2 \mathrm{~Hz}, \mathrm{H} 5 \mathrm{a}) ; 4.16(\mathrm{dd}, 1 \mathrm{H}, J=2.7,13.2 \mathrm{~Hz}, \mathrm{H} 5 \mathrm{~b}) ; 5.10(\mathrm{~d}, 1 \mathrm{H}, J=3.5 \mathrm{~Hz}, \mathrm{H1})$; $5.22(\mathrm{dd}, 1 \mathrm{H}, J=1.0,2.7 \mathrm{~Hz}, \mathrm{H} 4) ; 6.01(\mathrm{~d}, 1 \mathrm{H}, J=3.5 \mathrm{~Hz}, \mathrm{H} 2) ; 7.19$ (br d, 2H, $J=8.6$ Hz, Ar-H); 7.41 (br t, 2H, $J=7.8 \mathrm{~Hz}, \mathrm{Ar}-\mathrm{H}) ; 7.55$ (br t, $1 \mathrm{H}, J=7.1 \mathrm{~Hz}, \mathrm{Ar}-\mathrm{H}) ; 7.71$ (br d, $2 \mathrm{H}, J=8.6 \mathrm{~Hz}, \mathrm{Ar}-\mathrm{H}) ; 7.92$ (br d, $2 \mathrm{H}, J=8.4, \mathrm{Ar}-\mathrm{H}) .{ }^{13} \mathrm{C} \mathrm{NMR}\left(125 \mathrm{~Hz}, \mathrm{CDCl}_{3}\right) 22.7$, 57.0, 63.3, 66.3, 96.2, 129.3, 129.4, 130.4, 130.8, 131.0, 134.0, 134.4, 138.0, 146.3, 146.5, 166.7. HRMS (TOF) calcd for $\mathrm{C}_{20} \mathrm{H}_{20} \mathrm{O}_{7} \mathrm{SNa}(\mathrm{M}+\mathrm{Na})^{+} 427.0827$, found 427.0813. 

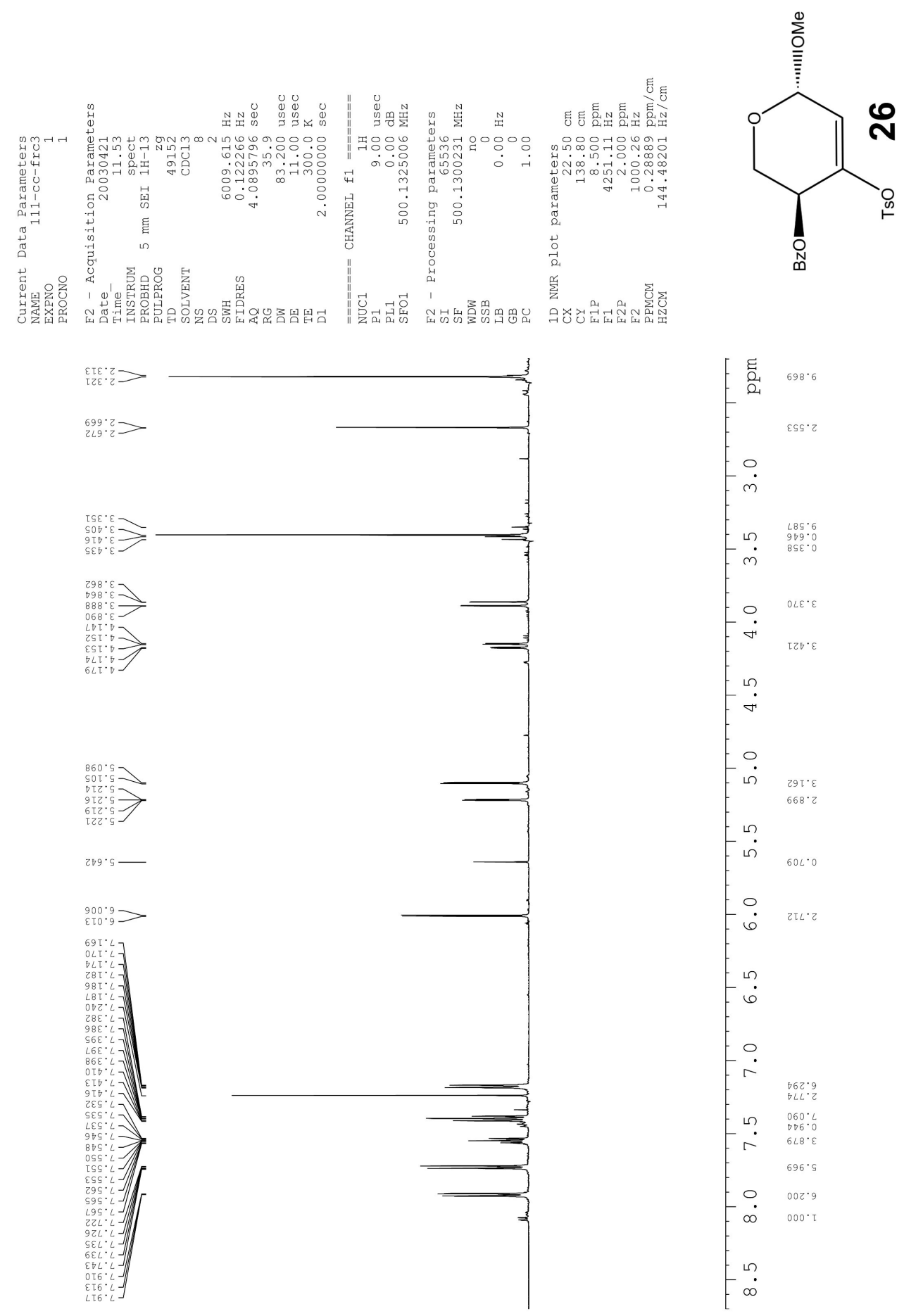

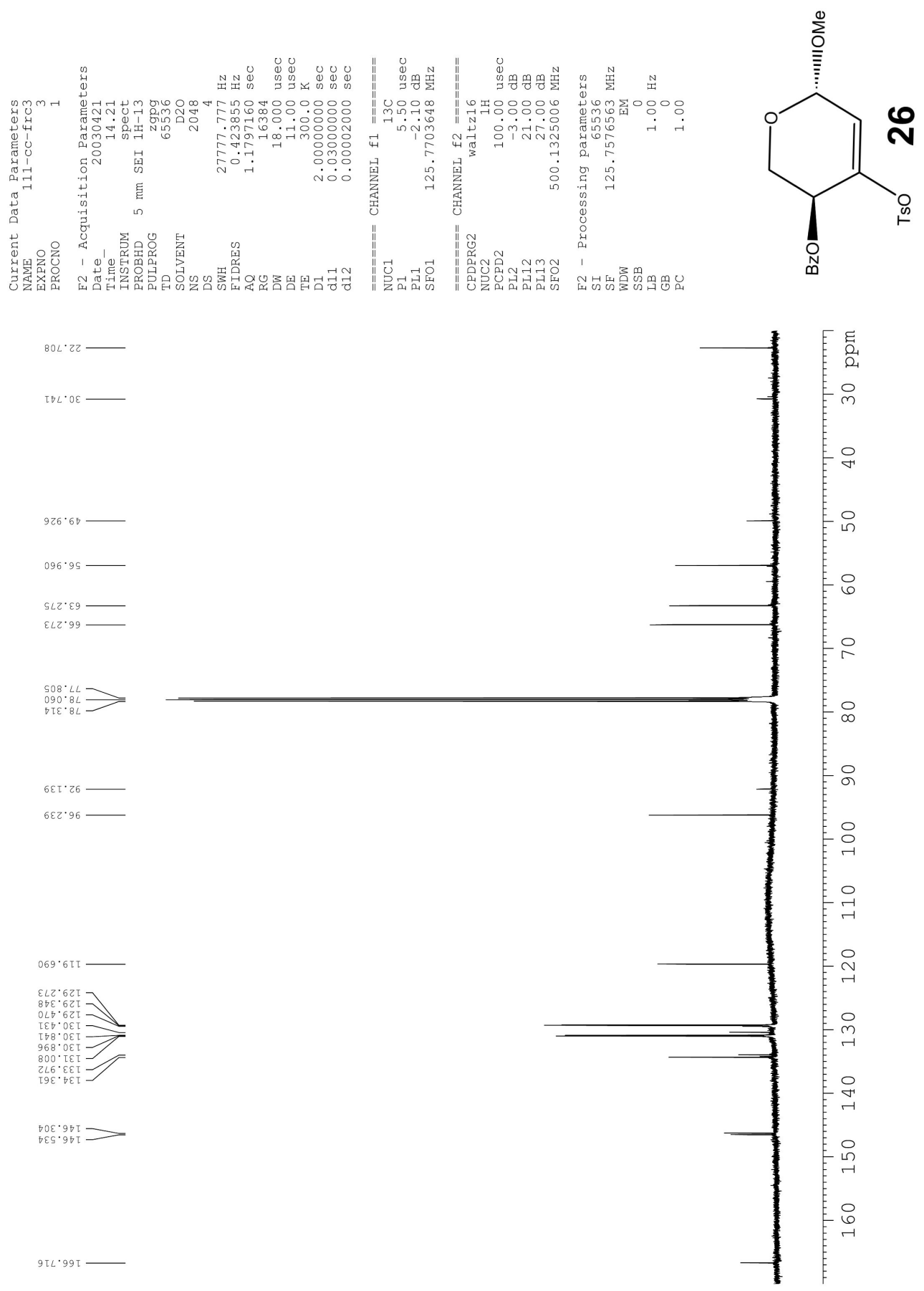


\section{References:}

(1) Indurugalla, D.; Bennet, A. J. Journal of the American Chemical Society 2001, 123, 10889-10898.

(2) McEwan, T.; McInnes, A. G.; Smith, D. G. Carbohydrate Research 1982, 104, 161-8.

(3) Jones, J. K. N.; Thompson, J. L. Canadian Journal of Chemistry 1957, 35, 955-9.

(4) Kondo, Y. Carbohydrate Research 1982, 110, 339-44.

(5) Dick, A. J.; Jones, J. K. N. Canadian Journal of Chemistry 1966, 44, 79-87.

(6) Buchanan, J. G.; Edgar, A. R. Carbohydrate Research 1976, 49, 289-304.

(7) Reeves, R. E. Journal of the American Chemical Society 1949, 71, 1737-9.

(8) Kondo, Y. Carbohydrate Research 1987, 162, 159-65.

(9) Kent, P. W.; Ward, P. F. V. Journal of the Chemical Society, Abstracts 1953, 41618. 